\title{
Single particle versus collectivity, shapes of exotic nuclei
}

Andrea Jungclaus

Instituto de Estructura de la Materia, CSIC, E-28006 Madrid, Spain

Received: date / Revised version: date

\begin{abstract}
In this article some selected topics of nuclear structure research will be discussed as illustration of the progress reached in this field during the last thirty years. These examples evidence the improvement of our understanding of the atomic nucleus reached on the basis of countless experiments, performed to study both exotic nuclei (nuclei far-off the valley of stability) as well as nuclei under exotic conditions (high excitation energy/temperature or large angular momentum/rotational frequency), using stable and radioactive ion beams. The experimental progress, in parallel to the advancement of modern theoretical descriptions, led us to a much richer view of this fundamental many-body system.
\end{abstract}

PACS. PACS-key discribing text of that key - PACS-key discribing text of that key

\section{Introduction}

During the last decades, nuclear structure physics has experienced a worldwide renaissance and our understanding of the atomic nucleus has not only improved, but very often we have found ourselves confronted with real surprises which demanded changes in our view of this fundamental system. As is often the case in the history of science, the very dynamic evolution of this field of research has been closely related to the development of new experimental tools. As we will see in the course of this article, the probably most important new tools were on one hand side the first generation radioactive ion beams and on the other hand highly efficient $4 \pi \gamma$-ray spectrometer in conjunction with different highly sophisticated ancillary detectors. In this article I will not try to provide an all-embracing view of nuclear physics research but instead illustrate the accomplished progress by means of some selected topics (evidently chosen according to personal taste). The focus will be on the "simple physics behind" and the qualitative understanding rather then detailed technical or theoretical aspects - assuming that many of these are presented in other contributions to this school.

Fig. 1 shows the chart of nuclides, the orientation map for nuclear physicists. The black squares mark the about 300 stable nuclei existing in nature and forming the valley of stability. The nuclei on the left side of this valley decay via $\beta^{+}$-decay, the ones on the right hand side via $\beta^{-}$-decay. Since the pioneering work by Maria Goeppert-Mayer and Hans Jensen in 1949 [1], we know that due to the shell structure of the atomic nucleus, the latter is particularly stable (has a large binding energy) for certain numbers of protons and neutrons, the so-called magic numbers. Nuclei in the vicinity of closed shells are spherical and their

Send offprint requests to:

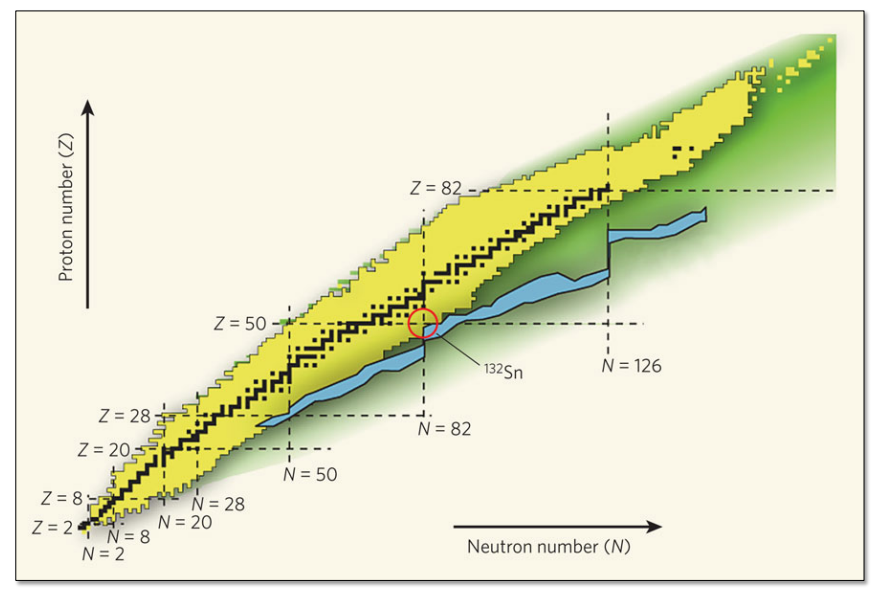

Fig. 1. Chart of nuclei with the black squares marking the about 300 stable nuclei existing in nature. The yellow area indicates the region of nuclei which already have been experimentally studied while the green region consists of thousands of nuclei which are expected to be bound (stable against particle emission) but have not yet been produced in terrestrial laboratories. Note that many of these nuclei are however continuously produced in the Universe during nucleosynthesis processes. The path of the astrophysical rapid neutron capture process is indicated in blue. Finally, the magic numbers of the nuclear shell model are included as black dashed lines.

excitation spectrum is dominated by single-particle excitations, whereas nuclei with many protons and neutrons outside the closed shells are very often deformed. In these nuclei collective excitations such as rotations or vibrations, to which many nucleons contribute, often become energetically favoured. The basic predictions of both the spherical shell model and the collective models have been confirmed in numerous studies in the past and it seemed 
that the atomic nucleus is - at least at not too high excitation energy and angular momentum and not too far off stability - very well understood.

In the last decades, however, it became possible to experimentally explore both exotic nuclei as well as nuclei under exotic conditions. By exotic nuclei, we understand nuclei far off stability, which includes i) nuclei with large isospin $\left(T_{3}=N-Z\right)$ close to the driplines and ii) superheavy nuclei, i.e. nuclei with large mass number $(A=N+Z)$. The driplines mark the limits of nuclear stability, i.e. outside these borders the nuclei are unstable against the emission of nucleons. In Section 2 of this article we will discuss how the first available energetic radioactive ion beams allowed to study light neutron-rich nuclei and to map the neutron dripline up to $Z=11$ and what we have learned from these studies. After that we move along the neutron-rich side of the chart of nuclides to slightly heavier systems and treat the evolution of nuclear shell structure at large isospin. Then we will discuss recent progress in the study of the properties of nuclei in the region around doublymagic ${ }^{132} \mathrm{Sn}$ and illustrate the relevance of the new results in view of the astrophysical rapid neutron capture process ( $r$ process) of nucleosynthesis. In Section 3 we move to the neutron-deficient side of the valley of stability and discuss topics such as the isospin symmetry in mediummass $N=Z$ nuclei, the contribution of $T=0$ isoscalar pairing to the properties of heavy $N=Z$ nuclei, the proton emission in nuclei close to, and in some cases even across, the proton dripline and finally the phenomenon of shape coexistence. To close the discussion of nuclei close to the limits of stability we will discuss in Section 4 the status of superheavy nucleus synthesis and close with remarks about the spectroscopy of transfermium nuclei.

Complementary to the investigation of exotic nuclei is the study of nuclei closer to stability, but under exotic conditions. In the last section of this article we will try to answer questions such as: How does a nucleus behave at high angular momentum and/or high excitation energies? Can a collapse of pairing correlations be observed under these circumstances? How does the shape of a nucleus change with angular momentum? What is the limit of deformation a nucleus can sustain? Finally, the article closes with a short outlook on the future of nuclear structure research.

\section{The neutron-rich side of the nuclear chart}

\subsection{Halos, skins and clusters in light nuclei}

The nuclear size and the proton and neutron density distributions are important bulk properties of nuclei that determine the nuclear potential, single-particle orbitals, and wave functions. Historically, the proton or charge distributions of stable nuclei have been studied using electron scattering, whereas for the measurement of the matter distributions strong interacting probes have to be used and elastic proton scattering provides the best information. From these studies, three basic properties have been established and can be found on the first pages of every nuclear physics textbook: i) the half-density radius of the matter distribution can be expressed as $R=r_{0} A^{1 / 3}$ with the radius constant $r_{0}=1.1-1.2 \mathrm{fm}$, ii) protons and neutrons are uniformly mixed in the nucleus, or in other words, there is no large difference observed in radii between the proton and neutron distributions and iii) the surface thickness is constant. The question we are going to discuss now is whether these three common properties of the nuclear density also hold for unstable nuclei.

Of course, we cannot produce targets of unstable nuclei to perform scattering experiments with electrons or protons. However, in the mid-eighties, first radioactive nuclear beams became available allowing for the determination of matter radii of the unstable nuclei from measured cross sections for their interaction and reaction with the nuclei of a stable target. In the pioneering work by Tanihata and co-workers [2], which was performed at the Lawrence Berkeley Laboratory (USA), the interaction cross sections between radioactive $\mathrm{Li}$ and $\mathrm{Be}$ beams and stable $\mathrm{Be}, \mathrm{C}$, and $\mathrm{Al}$ targets have been measured. For the first time, a sudden increase of the square mean radius has been observed within the Li chain of isotopes, in particular between ${ }^{9} \mathrm{Li}$ and the next bound isotope ${ }^{11} \mathrm{Li}$. This observation has been interpreted soon later as first evidence of a neutron halo in ${ }^{11} \mathrm{Li}$ by Hansen and Johnson [3]. Meanwhile, nuclei radii have been measured for numerous light unstable neutron-rich nuclei using the same technique. The results are summarized in a schematic way in Fig. 2. It is immediately obvious from this figure that the $R=r_{0} A^{1 / 3}$ rule is not valid at all far off stability, at least in the region of light nuclei. In contrary, large variations in radii are observed. Marked in this figure are the cases of well established $1 \mathrm{n}$ and $2 \mathrm{n}$ halos and the so far only known case of a proton halo in ${ }^{8} \mathrm{~B}$. Whereas in the case of ${ }^{11} \mathrm{Li}$ the radius changes drastically from one isotope to the next, a gradual increase of the matter radius is observed in the chains of heavier $\mathrm{F}, \mathrm{Ne}$ and $\mathrm{Na}$ isotopes. This phenomenon is understood as a neutron skin, which is an excess of neutrons at the nuclear surface. Generally it is not trivial to determine the radii of the proton and neutron distributions separately. An estimate of the charge radius can be obtained from the cross sections for charge-changing reactions at relativistic energies. However, a unique opportunity for a precise comparison between matter and charge radii over a wide range of neutron numbers is offered by the $\mathrm{Na}$ isotopes, since their rms charge radii have been determined in isotopeshift measurements [4]. The matter radii were determined as described above [5] and the resulting proton and neutron radii are shown in Fig. 2. We can see a gradual growth of the thickness of the neutron skin for neutron-rich $\mathrm{Na}$ isotopes up to $0.4 \mathrm{fm}$. It should be mentioned that additional information about the halo structure of neutron-rich nuclei, e.g. the wave function and density distribution of the valence nucleons, can be obtained from the measurement of the momentum distribution of fragments after their break-up (see also Section 2.3) or the cross sections for Coulomb break-up reactions. Narrow momentum distributions of fragments as well as large Coulomb break-up 


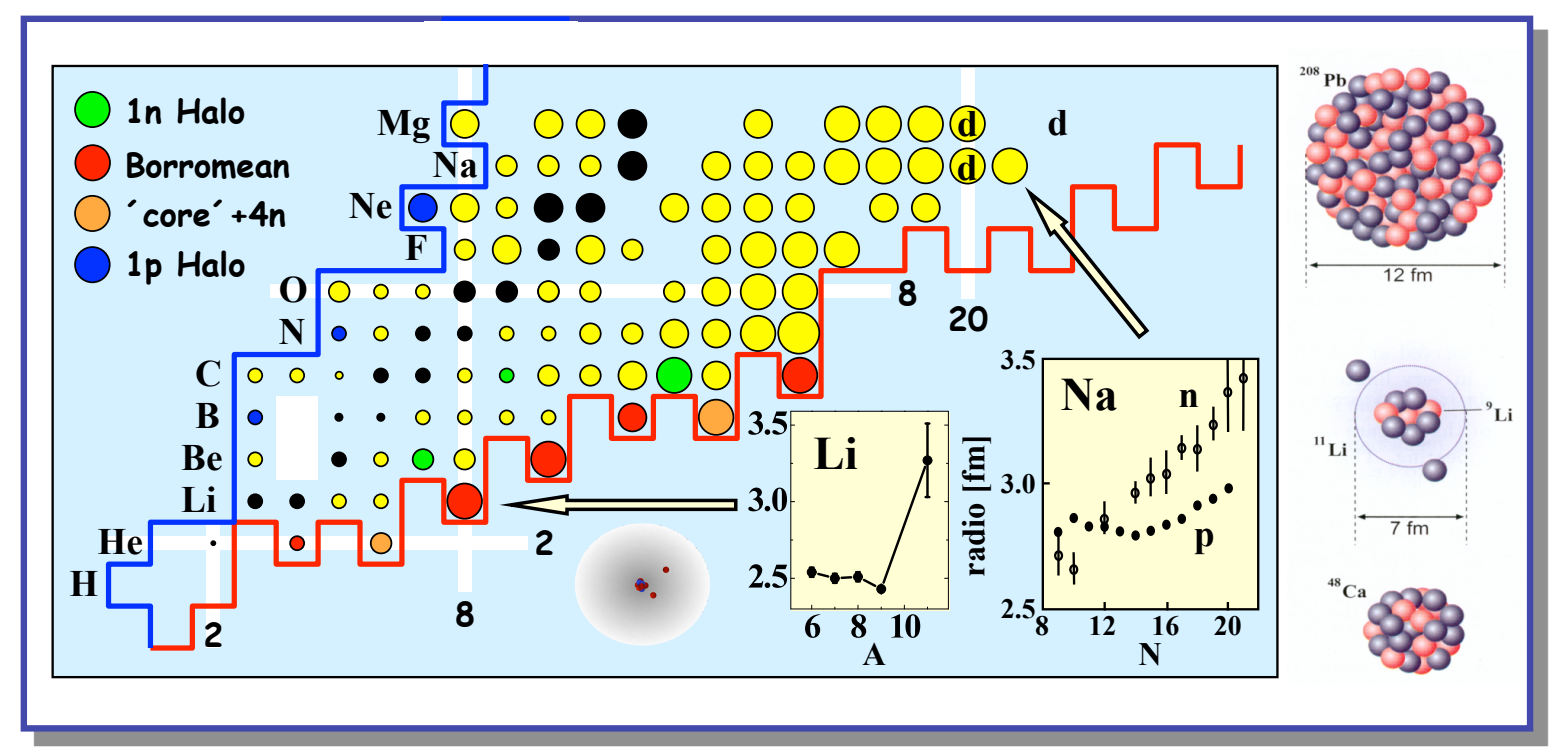

Fig. 2. Schematic summary of experimental quadratic mean square radii. The six different circle sizes correspond to the range of 2.0-3.5 fm. The experimental driplines are shown in red (neutrons) and blue (protons). In the lower part the mean square quadratic radii of the $\mathrm{Li}$ and $\mathrm{Na}$ isotopes (in the latter case separately for protons and neutrons) are shown while on the right hand side of the figure the sizes of some selected nuclei are illustrated.

probabilities indicate a relatively large spatial extent of the valence nucleon(s) [6,7]. A very nice review of the experimental status of nuclear halo structure studies is given in Ref. [8].

\subsection{Mapping of the neutron dripline}

The existence of a given nucleus is one of the most basic questions nuclear physics must answer. Therefore, aside from the study of the matter and charge distributions of neutron-rich light nuclei, already the mapping of the neutron dripline by itself is of major importance. One reason is that it allows to prove different mass predictions, another that shell effects should influence the nuclear binding and therefore the exact position of the dripline. Many experiments performed to map the dripline are very similar to the ones described in the last section. The neutronrich nuclei are produced in projectile fragmentation and identified using fragment recoil separators. As example, the particle identification plots obtained using the RIPS spectrometer at RIKEN (Tokyo, Japan) and the fragmentation of a) a $94.1 \mathrm{MeV} / \mathrm{u}{ }^{40} \mathrm{Ar}$ beam and b) a ${ }^{48} \mathrm{Ca}$ beam at $64 \mathrm{MeV} / \mathrm{u}$ are shown in Fig. $3[9,10]$. From this figure, it is immediately evident that the $N=2 Z+4$ nucleus ${ }^{31} \mathrm{~F}$ exists, whereas no events corresponding to ${ }^{28} \mathrm{O}$ and ${ }^{25} \mathrm{~N}$ have been observed. In the same way, the $N=2 Z+2$ isotope ${ }^{26} \mathrm{O}$ has not been detected though the heavier $N=2 Z+2$ nuclei ${ }^{29} \mathrm{~F}$ and ${ }^{32} \mathrm{Ne}$ could clearly be identified. Note, that none of the $N=2 Z+3$ nuclei in the range $5 \leq \mathrm{Z} \leq 10$ has been observed. The current knowledge of the neutron dripline obtained from this kind of experiments is summarized in Fig. 3. The most puzzling observation is that the heaviest
Nitrogen and Oxygen isotopes are ${ }^{23} \mathrm{~N}$ and ${ }^{24} \mathrm{O}$ with the same neutron number, $N=16$, while the heaviest isotope of fluorine observed so far is ${ }^{31} \mathrm{~F}$ with $N=22$. It is very astonishing that six additional neutrons can be bound by moving from Oxygen to Fluorine by adding just one proton. This location of the dripline for $\mathrm{O}$ and $\mathrm{F}$ is hardly predicted by mass formulas, most of which favor ${ }^{26} \mathrm{O}$ and ${ }^{29} \mathrm{~F}$ as heaviest isotopes. Fig. 3 shows that above $Z=9$, the dripline follows a more regular path as expected from mass formulas. The next $N=2 Z+4$ isotopes behind ${ }^{31} \mathrm{~F}$, namely ${ }^{34} \mathrm{Ne}$ and ${ }^{37} \mathrm{Na}$, are bound, too. Our current knowledge of the neutron dripline is limited to $Z \leq 11$ and one of the major goals for the future is to extent it to the heavier region. A first step in this direction constitutes the discovery of ${ }^{40} \mathrm{Mg}$ and ${ }^{42} \mathrm{Al}$ which was recently presented by Baumann et al. [11].

Let us come back now to the unexpected position of the dripline for the $\mathrm{O}$ and $\mathrm{F}$ nuclei. The sudden change in stability from oxygen to fluorine indicates an extra push of stability for the very neutron-rich fluorine isotopes. Where might this extra stability come from? One hint is given by the fact that among all the mass formulas on the market only the finite-range droplet model predicts the stability of ${ }^{31} \mathrm{~F}$ (and also ${ }^{31} \mathrm{Ne}$ ). This model includes nuclear deformation effects so one possible origin of the anomaly could be related to ground state deformation. Both ${ }^{31} \mathrm{Ne}$ and ${ }^{31} \mathrm{~F}$ with $N=21$ and $N=22$ are quite close to the $N=20$ shell closure of the spherical shell model. So one may ask whether the magic number $N=20$, implying closed shells and sphericity for nuclei close to the valley of stability, is still magic far off stability. This brings us directly to our next topic. 


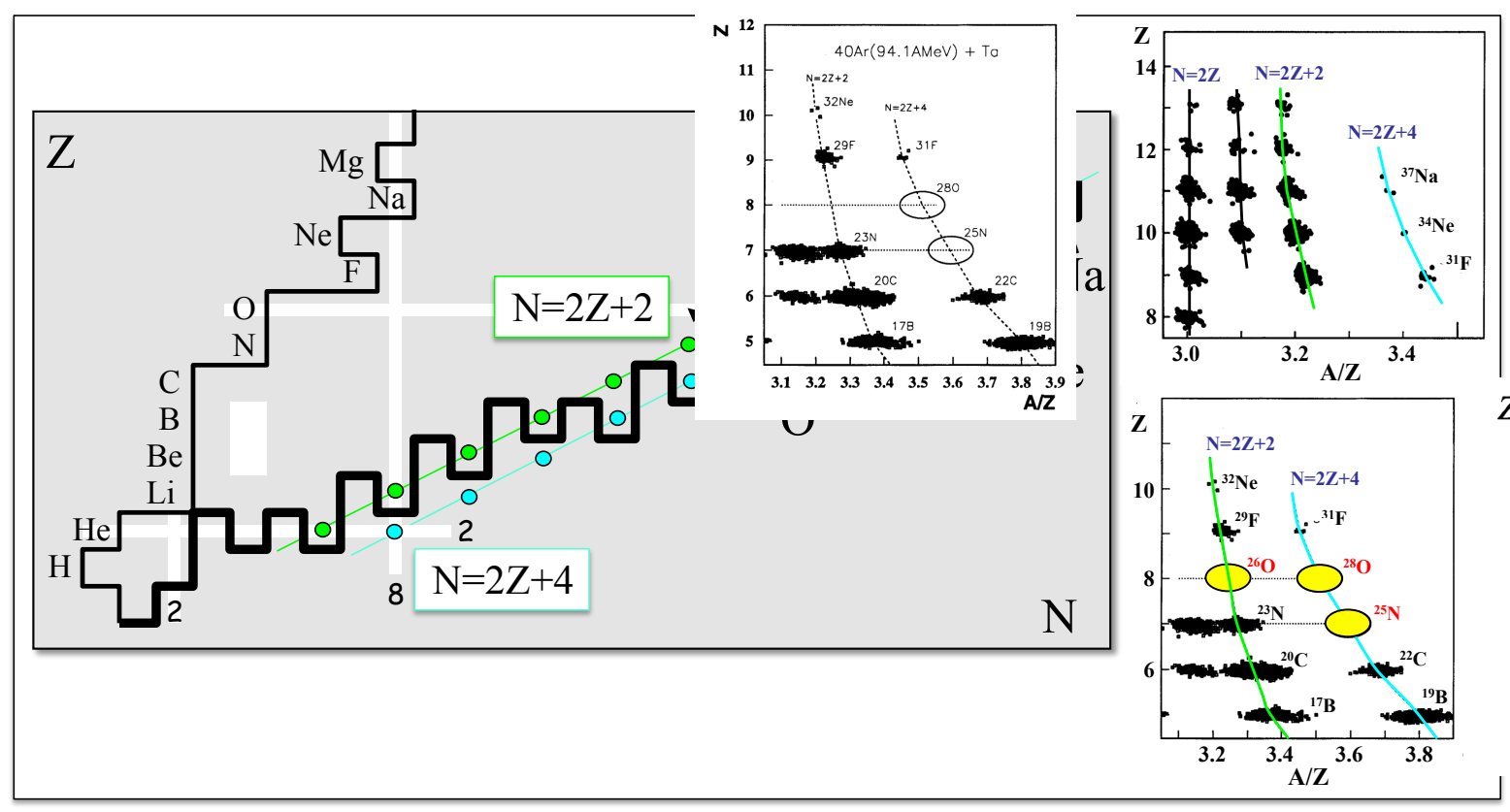

Fig. 3. Experimental neutron dripline in light nuclei up to $\mathrm{Na}$ illustrating the anomaly in its path for oxigen and fluorine isotopes. The insets show particle identification plots $(Z$ vs. $A / Z)$ obtained in two experiments performed at RIKEN employing the fragmentation of ${ }^{40} \mathrm{Ar}$ and ${ }^{48} \mathrm{Ca}$ beams $[9,10]$. In these plots each blob corresponds to a different isotope. A non-observation of a certain isotope implies that it is unbound (if it is not simply due to a lack of statistics).

\subsection{Shell evolution close to the neutron dripline}

In this article we will only present a short summary of the historic developments and the present status of our knowledge with respect to the evolution of the nuclear shell structure in the regions far-off the valley of stability. A more detailed discussion is not only outside the scope of the present article but also dispensable considering the large number of review articles covering this topic. Quite general information can be found in Refs. [12-15].

One of the first experimental indications for changes in the shell structure in neutron-rich nuclei dates back to 1975 when the masses of the Na isotopes were measured by Thibault et al. at ISOLDE (CERN, Switzerland) [16]. The results showed that the $N=20,21$ isotopes ${ }^{31,32} \mathrm{Na}$ "are more tightly bound than expected from theoretical predictions". Four years later, the first $2^{+}$state in ${ }^{32} \mathrm{Mg}_{20}$ was populated for the first time in the $\beta$ decay of ${ }^{32} \mathrm{Na}$ [17]. Its low excitation energy of $0.885 \mathrm{MeV}$, more than $2.4 \mathrm{MeV}$ lower as compared to the corresponding energies in the heavier $N=20$ isotones ${ }^{34} \mathrm{Si}$ and ${ }^{36} \mathrm{~S}$, clearly demonstrated a change of structure along the $N=20$ isotonic chain towards the neutron dripline. On the theoretical side, the first explanation for the experimental findings was presented by Warburton et al. within the framework of the nuclear shell model [18]. In that work it was suggested that in ${ }^{32} \mathrm{Mg}$ and some other nearby nuclei the deformed two-particle-two-hole ( $2 \mathrm{p}-2 \mathrm{~h})$ configuration, comprising the excitation of a neutron pair across the $N=20$ shell gap, would become energetically favoured and thus form the ground state. This inversion in excitation energy between the spherical $0 \mathrm{p}-0 \mathrm{~h}$ and the deformed $2 \mathrm{p}-2 \mathrm{~h}$ con- figurations lead to the denomination island of inversion for the region around $N=20$, in which the deformed $2 \mathrm{p}-2 \mathrm{~h}$ configuration forms the ground state. In the last decades numerous experiments, dedicated to the measurement of quantities such as electromagnetic transition strength and moments and employing a number of different reactions using slow and fast radioactive ion beams, allowed to determine the extent of the island of inversion, which is schematically indicated in Fig. 4, and construct a detailed and consistent picture of the structural changes taking place in this region of the nuclear chart.

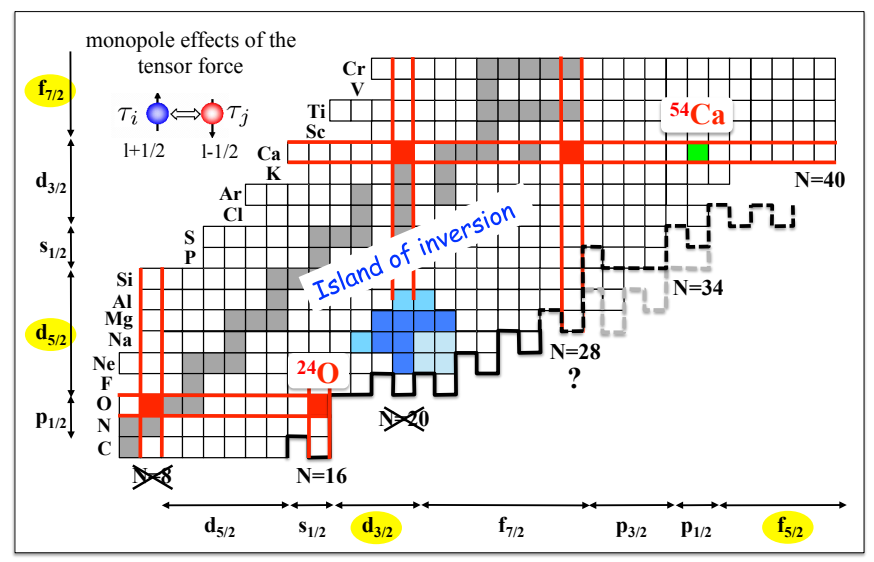

Fig. 4. Part of the chart of nuclides illustrating the shell evolution on the neutron-rich side close to the neutron dripline (see text for details). 
Later, the Monte Carlo shell model studies of the Tokyo group revealed the importance of the $T=0$ monopole interaction between the protons and neutrons for the description of the experimental findings $[19,20]$. This interaction is strongly attractive between orbital pairs with $j=l+1 / 2$ and $j=l-1 / 2$ while it is repulsive whenever the two nucleons both populate orbitals with either $j=l+1 / 2$ or $j=l-1 / 2$. These so-called monopole effects of the tensor force are responsible not only for the shell evolution around $N=20$ but also in other regions of the nuclear chart as we will see later. In the case of the island of inversion, the removal of protons from the $d_{5 / 2}$ orbital when moving from ${ }^{34} \mathrm{Si}$ down along the $N=20$ line towards ${ }^{28} \mathrm{O}$ pushes the neutron $d_{3 / 2}$ orbital up in energy (less proton-neutron pairs in the $j=l \pm 1 / 2$ orbitals $d_{3 / 2}$ and $\left.d_{5 / 2}\right)$. When the $d_{3 / 2}$ approaches the $f_{7 / 2}$ orbit above, the $N=20$ gap disappears and a new gap opens between $s_{1 / 2}$ and $d_{3 / 2}$, i.e. for $N=16$. Consequently, in this picture ${ }^{24} \mathrm{O}$ is expected to be a new doubly-magic nucleus. Experimentally, the magicity of this nucleus was demonstrated by Kanungo et al. in 2009 by measuring the longitudinal momentum distribution of one-neutron removal from ${ }^{24} \mathrm{O}$ [21]. The measured momentum distribution is very well described assuming that the last neutron occupies the $s_{1 / 2}$ orbital, no contribution from the $d_{3 / 2}$ orbit is observed. This finding clearly indicates the existence of an energy gap between these two orbitals, i.e. at $N=16$. Note that this scenario is in contrast to the one observed for ${ }^{11} \mathrm{Li}$ ten years before. In that case, Simon et al. found that the measured momentum distribution can only be described assuming a roughly equal occupancy of the $p_{1 / 2}$ and $s_{1 / 2}$ neutron orbitals [22]. This has been at that time the first indication of a vanishing $N=8$ shell gap away from stability, since the existence of a significant gap would prevent the neutron from occupying the $s_{1 / 2}$ orbital above the shell closure (see Fig. 5).

Inspection of Fig. 4 shows that the neutron-rich Ca isotopes offer an ideal opportunity to test the validity of the picture discussed above, namely the idea that monopole effects of the tensor force are responsible for the disappearance of the $N=20$ magic number in the island of inversion and the opening of a new shell gap at $N=16$. While the attractive interaction between protons and neutrons in the spin-orbit partner orbitals $d_{3 / 2}$ and $d_{5 / 2}$ played a major role there, the same picture should apply to the next spin-orbit pair, namely the $f_{5 / 2}$ and $f_{7 / 2}$ orbitals. The removal of protons from the $f_{7 / 2}$ orbit when going from stable ${ }^{62} \mathrm{Ni}$ down to ${ }^{54} \mathrm{Ca}$ should then reduce the binding of the $f_{5 / 2}$ proton orbital, therefore increase its energy and consequently lead to the development of a subshell gap for $N=34$ (compare Fig. 4). The trueness of this prediction has recently been investigated experimentally using in-beam $\gamma$-ray spectroscopy and proton knock-out reactions at relativistic energies at RIKEN [23]. In that work the excitation energy of the first excited $2^{+}$state in ${ }^{54} \mathrm{Ca}$ was measured and, in comparison to state-of-the-art shell model calculations, interpreted as "direct experimental evidence for the onset of a sizable sub-shell closure at neutron number 34 in isotopes far from stability". Note, however, that the evolution of the relative positions of the

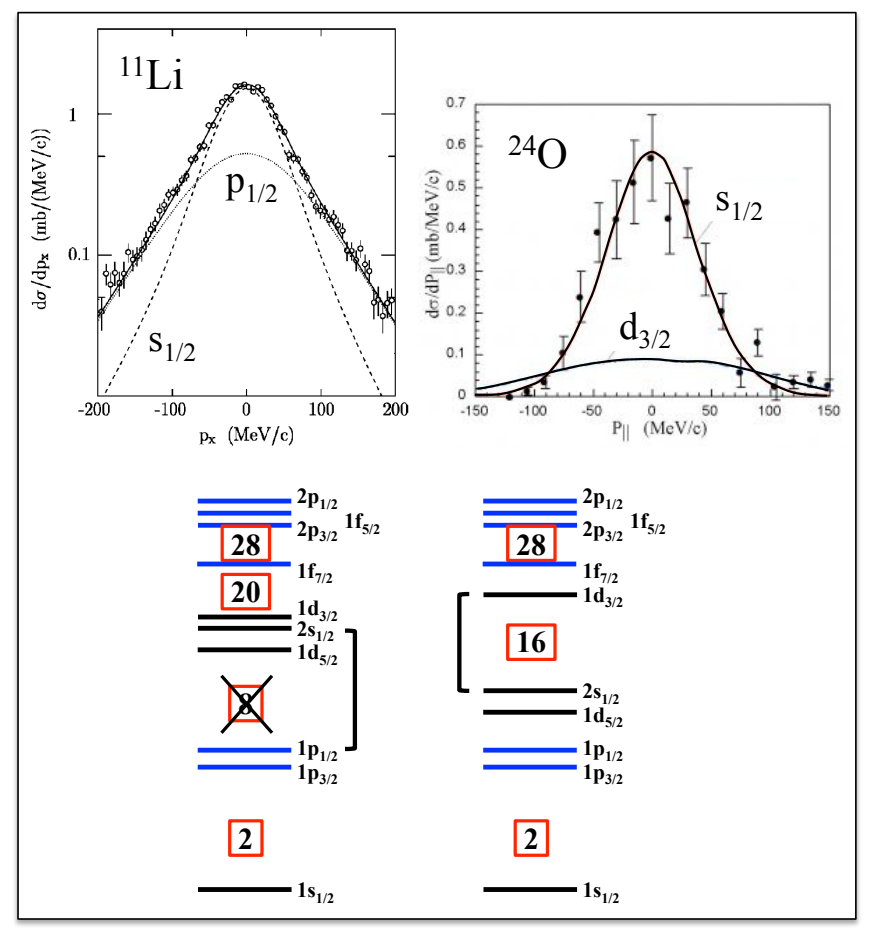

Fig. 5. Top: Measured longitudinal momentum distributions of one-neutron removal from ${ }^{11} \mathrm{Li}$ and ${ }^{24} \mathrm{O}$. The data are daten from Refs. $[22,21]$. The bottom panel illustrates the consequences of the measured momentum distributions for the evolution of shell structure.

different orbitals depends on a variety of different subtle effects, not only the monopole term of the tensor force. Whether the size of the $N=34$ gap is really large enough to call $N=34$ a new magic number is in my opinion not so clear. In another Nature article [24], published just four months before Ref. [23], mass measurements were presented which "unambiguously establish a prominent shell closure at neutron number $N=32$ ". Note, that $N=32$ and $N=34$ are only separated by the $p_{1 / 2}$ orbital!

So far we discussed the evolution solely within the framework of the nuclear shell model and tried to understand the experimental observation using simple pictures. We would like to mention, however, that besides the SM there is a second main theoretical approach to study the structure of the atomic nucleus, namely the mean field theory and beyond. The basic mean field approach is the Hartree-Fock-Bogolibov (HFB) in which the long range part of the nuclear interaction (particle-hole) and the short range part (particle-particle) are treated at the same foot and allows a good description of deformations and pairing properties. As a matter of fact the HFB approach, in conjunction with density dependent interactions like Skyrme and Gogny, is well known since long ago for providing a good description of nuclear bulk properties like binding energies, quadrupole moments, pairing gaps, radii etc, all over the periodic table. The Gogny force in particular has a large predicting power. Recently there has been two main advances in the mean field approaches [25]. First, by the recovery of the symmetries, like the particle 
number and the angular momentum broken in the HFB approach, by the projection technique. And second, by including fluctuations, around the most probable values of the HFB theory, by the Generator Coordinate method (GCM) like the $(\beta, \gamma)$ deformations [26], the pairing gaps $\left(\Delta_{Z}, \Delta_{N}\right)[27]$ and very recently the angular frequency $(\hbar \omega)$ [28]. The combination of these two developments in the so called Symmetry Conserving Configuration Mixing Approach (SCCMA) provides the description of nuclear spectroscopic properties with a high accuracy. Furthermore the use of a single interaction along the nuclide chart with a good description of bulk properties, provides a large predicting power to these approaches. The examples of shell evolution discussed above are discussed in the framework of beyond mean field theory in Refs. [28-30].

Moving from the disappearance of the $N=20$ magic number and the simultaneous emergence of a shell gap at $N=16$ to the case of a potential new $N=34$ sub-shell closure, we skipped the discussion of a possible alteration of the $N=28$ magic number. This is the first of the classical magic numbers which originates from the spin-orbit interaction. Although in contrast to the case of $N=20$ the dripline has not yet been reached experimentally for $N=28$, numerous experiments have evidenced modifications of this shell gap in the very neutron-rich isotope ${ }^{42} \mathrm{Si}$ [31-33]. The results pinpoint the effects of several terms of the nucleon-nucleon interaction, besides the already discussed tensor component, namely the central, the spinorbit and the three-body contributions. All these aspects are discussed in detail in Ref. [15] so that it is not necessary nor beneficial to repeat it here. For the larger magic numbers, namely 50, 82 and 126, the neutron dripline is still far out of reach experimentally and theoretical calculations predicting changes in the shell structure of heavy neutron-rich nuclei are therefore difficult to test. In particular, the evolution of the $N=82$ shell gap has been subject of numerous theoretical studies in the past since it is of utmost importance for the exact course of the astrophysical rapid neutron capture process ( $r$ process) of nucleosynthesis. To which extent experimental information can contribute to the better understanding of such fundamental processes even without reaching the limits of nuclear binding, i.e. the dripline, will be discussed in the next section.

\subsection{The region around doubly-magic ${ }^{132} \mathrm{Sn}$ and the $r$ process of nucleosynthesis}

The origin of the heavy elements from iron to uranium is one of the main open questions in science. The slow neutron-capture process ( $s$ process) of nucleosynthesis, occurring primarily in helium-burning zones of stars, produces about half of the heavy element abundance in the Universe. The remaining half requires a more violent process, the $r$ process. During the $r$ process, in environments of extreme temperatures and neutron densities, a reaction network of neutron captures and $\beta$ decays synthesizes very neutron-rich isotopes in a fraction of a second. These isotopes, upon exhaustion of the supply of free neutrons, de- cay into the stable or semistable isotopes observed in the solar system. However, none of the proposed stellar scenarios, including the explosion of supernovae and merging neutron stars, can fully explain the available abundance observations. Also the mechanism of the $r$ process is uncertain. Nuclear physics properties such as $\beta$-decay halflives and masses are key for predicting abundance patterns and thus extracting signatures of the $r$ process from a detailed comparison to astronomical observations. The nuclei below doubly-magic ${ }^{132} \mathrm{Sn}$, i.e. the ones with proton number $Z \leq 50$ and neutron number $N \sim 82$, are crucial in any $r$-process mechanism because their enhanced binding bends the $r$-process path closer to stability (compare Fig. 1) slowing down the reaction flow. The half-lives of these so-called waiting-point nuclei determine the time scale of the $r$ process and shape the prominent $r$-process abundance peak of isotopes with $A \sim 130$. The precise theoretical prediction of these half-lives is challenging because the structure evolution of neutron-rich $N \sim 82$ nuclei is still unknown.

In a recent experiment performed at the RIBF facility at RIKEN the half-lives of 110 neutron-rich nuclei across the $N=82$ shell gap were measured, 40 of them for the first time [34]. The implications of these new half-lives for the $r$ process were investigated by conducting a fully dynamic reaction-network calculation study that simulated a spherically symmetric outflow from a neutron-rich stellar environment. The impact of the new measurements on the calculated solar-abundance pattern is illustrated in Fig. 6, where two calculations are compared that differ only by the use of the newly measured half-lives instead of theoretical values. All the other nuclear structure data, as well as the astrophysics conditions, were the same. The new half-lives have a global impact on the calculated $r$-process abundances, and alleviate the underproduction of isotopes just below and above the $A \sim 130$ peak, which in the past required the introduction of shell-structure modifications $[35,36]$. A particularly beneficial effect of the new half-lives of the most neutron-rich isotopes of $\mathrm{Ag}, \mathrm{Cd}$, In, and $\mathrm{Sn}$ $(N>82)$, is to greatly improve the description of the abundance of rare earth elements, which is a prerequisite for studying the universality of the $r$ process. The reduced nuclear physics uncertainty provides a new level of reliability for $r$-process calculations and their parametrized astrophysical conditions.

\section{The neutron-deficient side of the nuclear chart}

While we have shown in Section 2 that most of the progress achieved during the last decades on the neutron-rich side of the nuclear chart is owing to the availability of lowand high-energy radioactive ion beams, on the neutrondeficient side the majority of nuclei up to the proton dripline can be studied using stable ion beams employing the fusionevaporation reaction, which is also called compound nucleus reaction. In this reaction two stable nuclei are brought together at an energy above the Coulomb barrier. The 


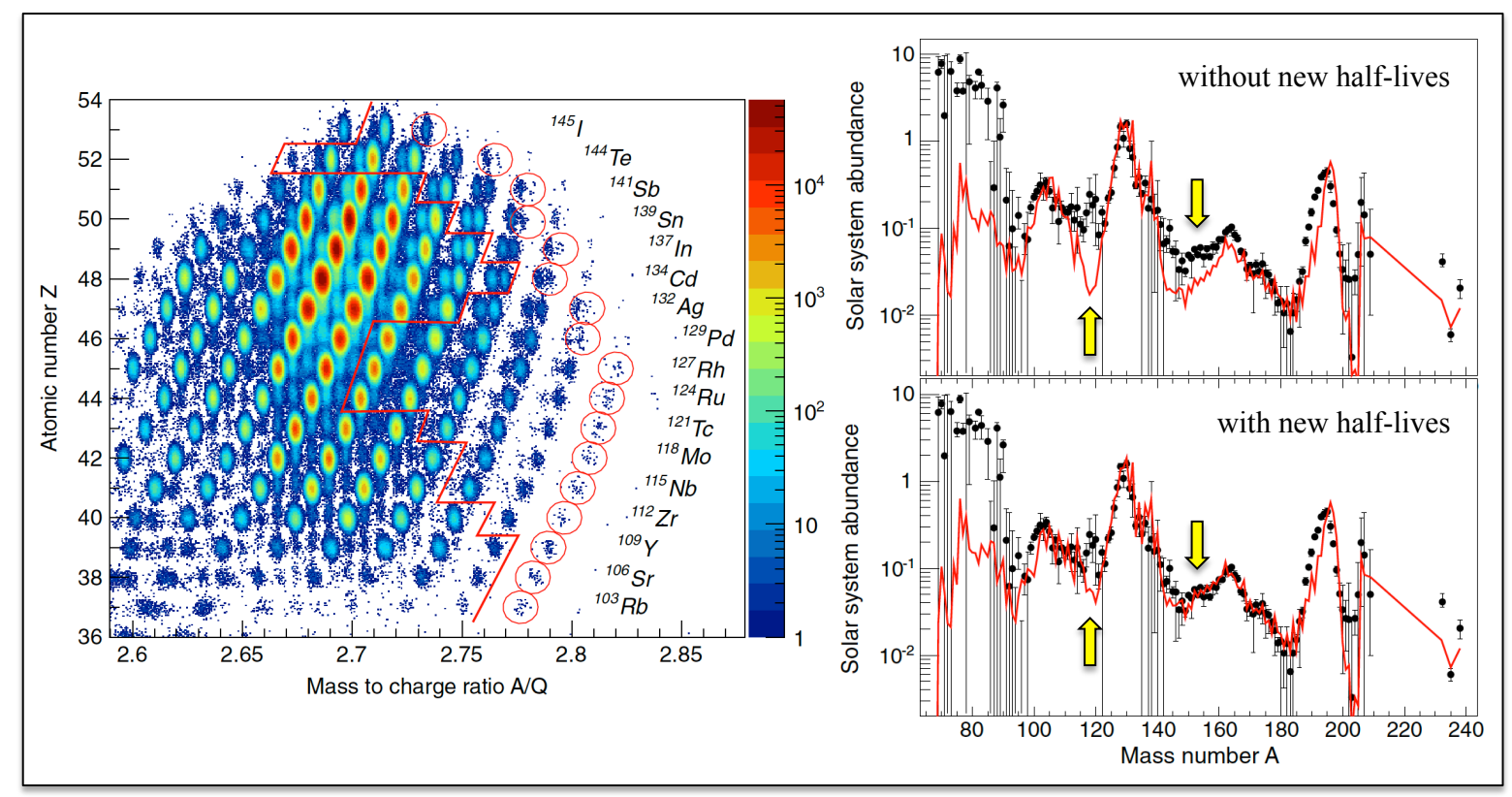

Fig. 6. Left: Particle identification plot showing all events according to the reconstructued values of the atomic mass, $Z$, and the mass-to-charge ratio, $A / q$. Nuclei with newly measured half-lives are on the right side of the red solid line. The heaviest masses for which half-lives could be measured are highlighted by red circles. Right: Comparison between the $r$-process solar abundance pattern and the abundances calculated without (top) and with (bottom) the new experimental half-lives. Figure adopted from Ref. [34].

formed system then either fissions immediately or a compound nucleus is formed in a highly excited state as illustrated in Fig. 7. The compound nucleus reaches statistical equilibrium and subsequently decays mainly by particle emission until this is no longer possible energetically. Alternatively, with a branching of $10^{-2}-10^{-4}$, the disexcitation also proceeds by high-energy $\gamma$ rays from the giant dipole resonance. Finally, the residual nuclei deexcite to the ground state via the emission of $\gamma$ radiation. In the time interval between the formation and the decay of the compound nucleus, the excitation energy is shared statistically among its constituent nucleons so that all memory of its mode of formation is lost. The processes of formation and decay are thus independent of each other. This reaction produces nuclei on the neutron-deficient side of the valley of stability because stable light or medium mass nuclei used as target and/or projectile have a lower $N / Z$ ratio as compared to heavy stable nuclei (see Fig. 1). Using this reaction a variety of physics topics has been studied in neutron-deficient nuclei in the past, for example the proton emission from ground and excited states, the isospin symmetry in $N=Z$ nuclei or the shape existence phenomenon. These topics will be discussed in the following subsections. Furthermore, the fusion-evaporation reaction is also the main tool to study the heavy end of the nuclear chart, i.e. to synthesize superheavy elements or to perform spectroscopy of transfermium nuclei (see Section 4). Finally, since this reaction allows to populate highly excited states with high angular momentum (see Fig. 7), it is also perfectly suited, in combination with highlyefficient $\gamma$-ray spectrometer, to investigate the behavior of the atomic nucleus under extreme conditions of excitation energy and rotational frequency. Phenomena which only arise under these conditions will be discussed in Section 5.

\subsection{Proton emitters and proton radioactivity}

The limits of stability, i.e. the driplines, are reached when the nuclear force is no longer able to bind an ensemble of nucleons with a too large neutron or proton excess. Whereas for heavy nuclei, $\alpha$ decay, ${ }^{14} \mathrm{C}$ radioactivity or fission occurs, unstable proton-rich (or neutron-deficient) nuclei may decay from their ground states by the emission of one proton in the case of odd- $Z$ nuclei or of two protons for even- $Z$ nuclei. The one-proton ground state decay was observed for the first time at GSI (Darmstadt, Germany) in $1981[37,38]$. In the meantime, more than 30 cases of proton radioactivity have been identified for odd$Z$ nuclei beyond the proton drip line between $Z=51$ and $Z=83$. This decay mode so far has only been observed for relatively heavy nuclei because here the presence of a large Coulomb barrier reduces the proton barrier penetration probability to the extent that proton decays of nuclei from their ground state have measurably long halflives. The proton decay rate depends both on the energy and orbital angular momentum of the emitted proton as well as on the wave function of the proton-decaying state, which is determined by the shape of the nuclear potential and by residual interactions between valence nucleons. The measurement of proton decay rates is therefore a 


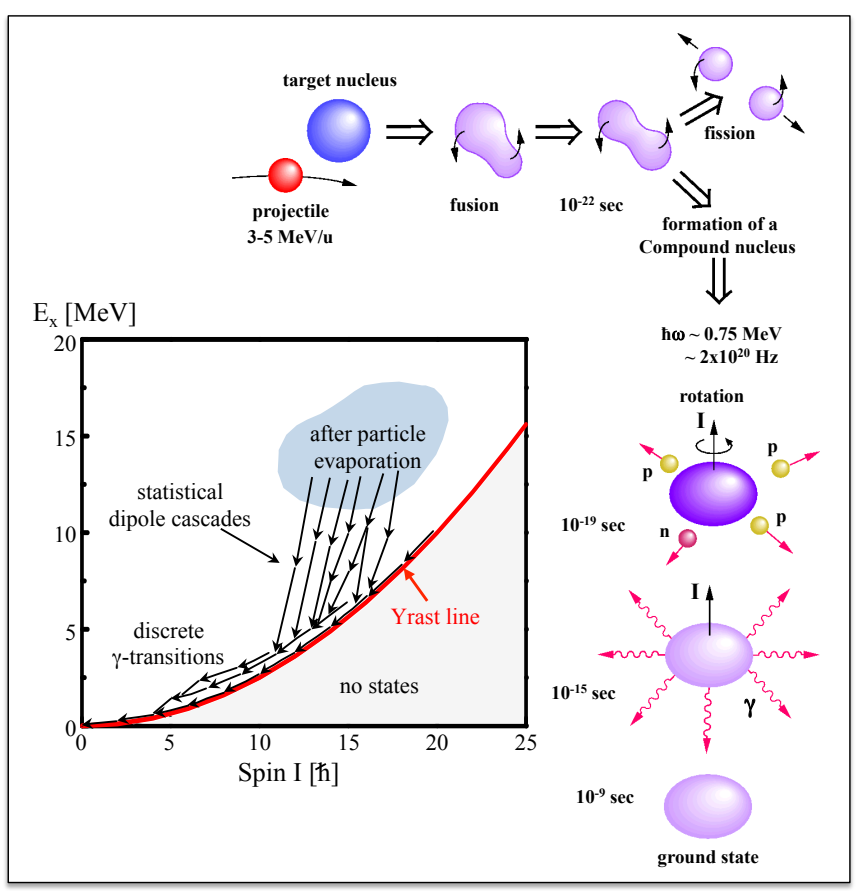

Fig. 7. Schematical illustration of the compound nucleus reaction. The inset shows the population of excited states of the reaction products in the excitation energy vs. spin plane.

unique tool to establish the sequence of shell-model singleparticle levels beyond the proton drip line. Whereas most of the known proton emitters have decay rates consistent with the assumption of a spherical potential, for some proton emitters in the rare earth region anomalous decay rates were observed indicating the onset of deformation. A more direct way to deduce information about the shape of a nucleus is of course the determination of its excitation spectrum. However, proton emitters are populated only with very small cross sections in heavy-ion induced fusionevaporation reactions with stable ion beams, ranging from hundreds of microbarns to tens of nanobarns. Furthermore, these rare events have to be identified in the presence of a background of hundreds of millibarns from the strong reaction channels. Therefore prompt $\gamma$-ray spectroscopy of proton emitters beyond the drip line became feasible only very recently employing the recoil-decay tagging method in combination with highly-efficient $4 \pi \gamma$-ray spectrometer (see Section 5). As one recent example, we will discuss the $\gamma$-spectroscopic study of the proton emitter ${ }^{141}$ Ho. This nucleus has been populated in the fusionevaporation reaction ${ }^{92} \mathrm{Mo}\left({ }^{54} \mathrm{Fe}, \mathrm{p} 4 \mathrm{n}\right)$ with a cross section of about $100 \mathrm{nb}$ [39], which has to be compared to the total reaction cross section in the order of $1 \mathrm{~b}$. That means that only in one out of 10,000,000 reactions between target and beam nuclei, ${ }^{141} \mathrm{Ho}$ is the reaction product. The trick of the recoil decay tagging technique is to correlate evaporation residues, which are unambiguously identified by their subsequent decay which takes place several $\mu$ s or even milliseconds after their production, with prompt $\gamma$-rays, detected immediately after the nuclear reaction took place.
Using this technique, it has been possible to observe rotational bands feeding both the ground state $\left(\mathrm{T}_{1 / 2}=4.2(4)\right.$ $\mathrm{ms})$ and a second proton-emitting state, namely an isomer at about $60 \mathrm{keV}$ excitation energy $\left(\mathrm{T}_{1 / 2}=8(3) \mu \mathrm{s}\right)[39]$. This observation constitutes direct evidence that ${ }^{141} \mathrm{Ho}$ is deformed. A quadrupole deformation of $\beta_{2}=0.25$ (4) was deduced for the ground state from the extracted dynamic moment of inertia. In a later study [40], a fine structure in the proton emission from both states was observed, indicating the population of the first $2^{+}$state in ${ }^{140}$ Dy with a branching ratio of $1-2 \%$, competing with the dominant branch to the $0^{+}$ground state.

Already in 1960 two-proton radioactivity has been predicted to occur for even-Z proton-rich nuclei beyond the dripline [41]. This process is very difficult to observe and only in 2002, two-proton radioactivity has been observed for the first time in ${ }^{45} \mathrm{Fe}[42,43]$, see below. However, already in 1983 the first case of $\beta$-delayed $2 \mathrm{p}$ emission was discovered [44] and up to now, several other cases are established. Beta-delayed multiparticle emission becomes increasingly important when approaching the drip lines because the $\mathrm{Q}_{\beta}$ values increase while the particle separation energies decrease [45]. In nearly all cases, the observed $\beta 2 \mathrm{p}$ originates from the Isobaric Analogue State in the daughter nucleus. It these studies, it has been possible to answer the question about the underlying decay mechanism. In principle, both a direct emission of two protons as well as a sequential decay via intermediate states is possible. Whereas in the latter case, the individual proton energies depend on the intermediate state and the proton energy spectrum therefore exhibits peaks at certain energies, this spectrum is continuous in direct emission. A second criterion comes from the angular distribution between the two protons. Whereas in the sequential case the angular dependence introduced by the momentum coupling is negligible, the angular distribution is far from being isotropic in direct emission. Using these two criteria, it has been shown that in all known cases of betadelayed two-proton emission the mechanism is sequential decay and it is therefore possible to derive information about excited states in the intermediate nucleus. No evidence of a non-sequential decay could be pointed out. However, if there is no accessible intermediate state, the sequential emission is forbidden and the emission of the two protons is simultaneous. This situation is expected for ground state decays of some even-Z nuclei, which due to the pairing energy are more bound than the one-proton daughter. Then two cases can be distinguished: First, the decay may proceed via a three-body disintegration. This has been observed for the two-proton decays of the broad resonance states of ${ }^{6} \mathrm{Be}[46]$ and ${ }^{12} \mathrm{O}$ [47]. Secondly, the nuclear state may decay via ${ }^{2} \mathrm{He}$ emission and a strong angular and energy correlation between the two resulting protons may be expected. The nucleus ${ }^{45} \mathrm{Fe}$ has been the first case for which two-proton radioactivity from a narrow nuclear ground state was observed in 2002, namely in two experiments performed at the same time at GSI [42] and GANIL [43]. In both cases projectile fragmentation of a ${ }^{58} \mathrm{Ni}$ beam was used to produce ${ }^{45} \mathrm{Fe}$, at $650 \mathrm{MeV} / \mathrm{u}$ at 
GSI and at a lower energy of $75 \mathrm{MeV} / \mathrm{u}$ at GANIL. The fragments were identified on the basis of flight times and energy losses and then stopped in position sensitive silicon detectors which allowed to observe their subsequent decays. The two-proton decay mode was unambiguously identified by measuring the decay energy and half-life and the observation of the daughter decay with a half-life in agreement with the half-life of ${ }^{43} \mathrm{Cr}$, the $2 \mathrm{p}$ daughter of ${ }^{45} \mathrm{Fe}$, and in disagreement with all other possible daughters (for details see $[42,43]$ ). Note that in these experiments as few as 5 and 20 decay events of ${ }^{45} \mathrm{Fe}$ were detected at GSI and GANIL, respectively. After these pioneering experiments, several other cases of two-proton radioactivity were observed. However, in none of these experiments, the two protons were identified separately. A real breakthrough was then achieved five years later, again nearly at the same time independently by two different research collaborations, employing a very powerful new technique. Instead of implanting the ${ }^{45} \mathrm{Fe}$ ions produced in fragmentation reaction in position sensitive silicon detectors, they were now implanted in time-projection chambers (TPC), which allow for a visualization in three dimensions of implantation and decay events and consequently for a direct observation of the individual protons. In an experiment performed at GANIL (Caen, France) a total of $10{ }^{45} \mathrm{Fe}$ were implanted in a TPC and correlated with successive decays [48]. At NSCL (MSU, USA) even 125 decays were observed, 87 of them followed by $2 \mathrm{p}$ emission and 38 by $\beta$ decay with successive proton emission [49]. In this experiment the ${ }^{45} \mathrm{Fe}$ ions were implanted in an optical TPC which allows to take photographs of the $2 p$ decays. An example of such an image recorded by a CCD camera is shown in Fig. 8. A track of a ${ }^{45} \mathrm{Fe}$ ion entering the chamber from the left is seen. The two bright, short tracks are protons of approximately $0.6 \mathrm{MeV}$, emitted $535 \mu$ s after the implantation. This single image is the most impressive proof for the existence of ground-state two-proton radioactivity as nuclear decay mode. The information from the optical TPC allows for a full reconstruction of the decay event in three dimensions. It is therefore possible to determine the opening angle between the two protons emitted in the decay of ${ }^{45} \mathrm{Fe}$. The distribution of this opening angle is a clear signature for the decay mechanism, three-body disintegration versus ${ }^{2} \mathrm{He}$ emission (see above). The measured distribution of this opening angle is shown in Fig. 8. A two-bumped structure is evident with one broad peak around $50^{\circ}$ and a smaller one around $145^{\circ}$. It is nicely reproduced by predictions of a model which includes explicitly the three-body dynamics of the decay process. On the other hand, a simple diproton scenario can be ruled out since in this case, a distribution containing only one narrow peak around $30^{\circ}$ is expected, in clear contradiction to the experimental finding.

A summary of the very impressive progress achieved in recent years in the study of radioactive decays at the limits of nuclear stability is presented in Ref. [50].

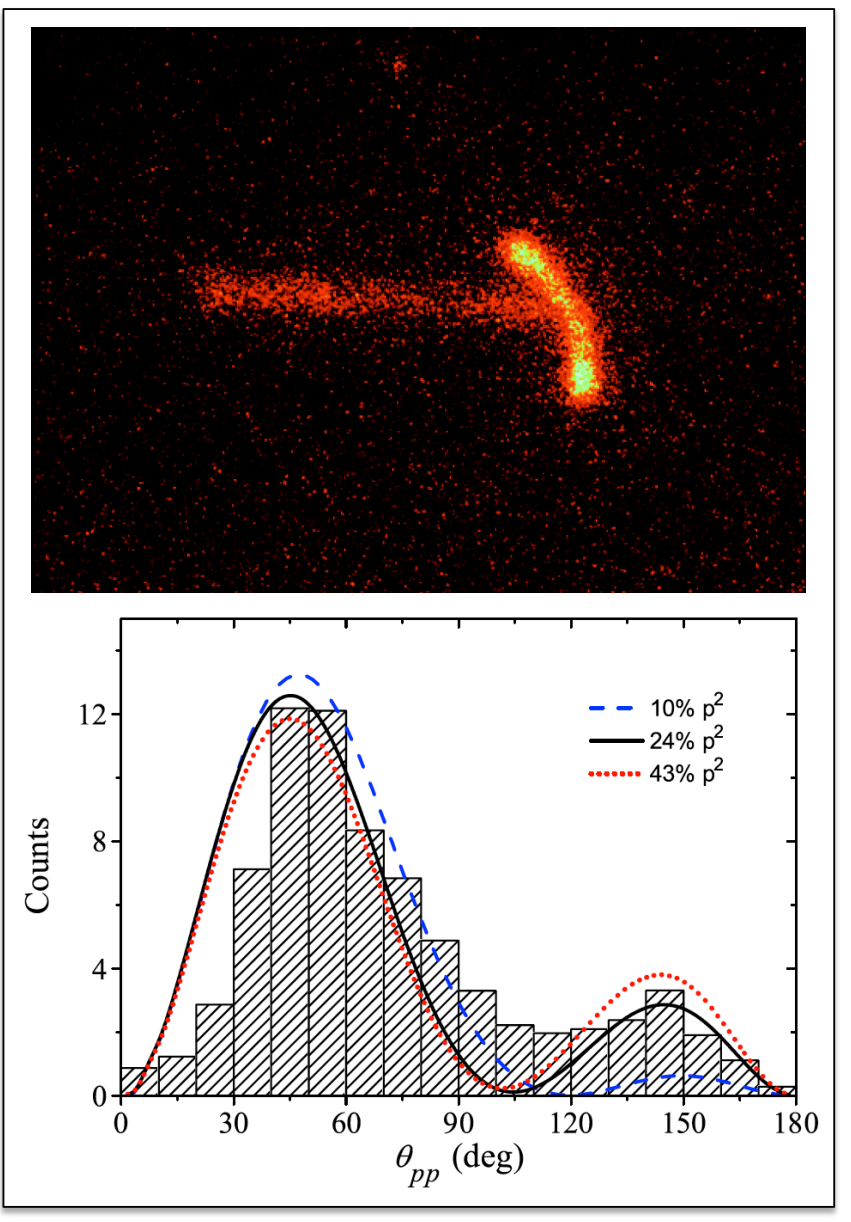

Fig. 8. Top: Image of a two-proton decay of ${ }^{45} \mathrm{Fe}$ registered by a CCD camera. The long track corresponds to the ion entering the TPC from the left while the two short tracks are the two protons emitted in the ground-state decay. Bottom: Measured distribution of the opening angle between the two protons emitted in the decay of ${ }^{45} \mathrm{Fe}$ (histogram) compared to predictions of a three-body model. Figure adopted from Ref. [49].

\subsection{Isospin symmetry studies in $N=Z$ nuclei}

The nuclei on, and around, the line of $N=Z$ between $A \sim 40$ and $A \sim 70$ have been the focus of enormous experimental effort in recent years. In this region, a rapid evolution of structure occurs when leaving the line of stability towards the proton dripline. This is also a region where state-ofthe-art shell-model calculations are available which can help to interpret experimental data. One of the most important aspects is that these nuclei allow to study one of the fundamental symmetries of modern physics, namely the neutron-proton exchange symmetry that leads to the concept of isospin in nuclear physics. Isospin symmetry is a consequence of the almost perfect charge-independence and charge symmetry of the attractive strong nucleonnucleon interaction. The symmetry is most evident when considering reflections along the $N=Z$ line, i.e. studying nuclei with exchanged numbers of neutrons and protons, the so-called mirror nuclei. As a case study we will discuss 


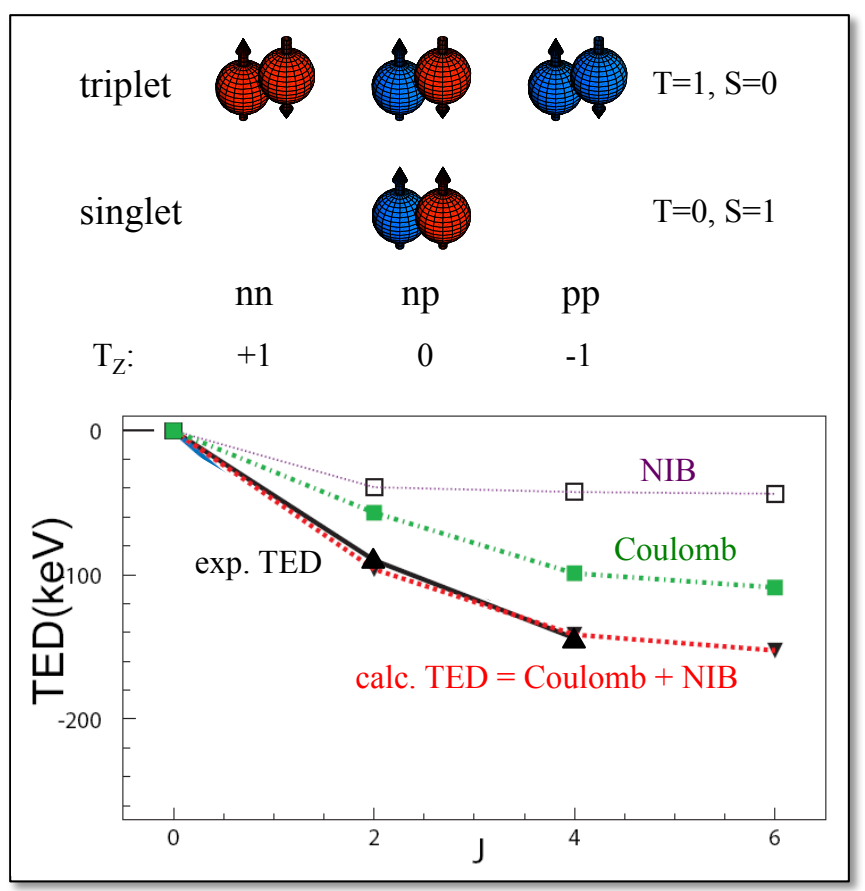

Fig. 9. Upper part: Illustration of the two different types of proton-neutron pairing, $T=0$ and $T=1$. Note that $T=0$ pairing is forbidden for proton-proton and neutron-neutron pairs. Lower part: Experimental triplet energy difference, TED, as a function of spin for the $A=54$ isobaric triplet. The experimental values (black triangles) are compared to the result of shell model calculations including both Coulomb and nuclear isospin-breaking (NIB) terms.

the example of the mirror pair ${ }^{54} \mathrm{Ni} /{ }^{54} \mathrm{Fe}$ with $28 / 26$ protons and 26/58 neutrons, respectively. These two nuclei have $T_{z}=-1,+1$, respectively, and the ground state structure of each nucleus has isospin $T=1$ (see Fig. 9). The sets of $T=1$ states in these two nuclei would be identical for a perfectly charge-symmetric nuclear force and in the absence of any other isospin-breaking effects. However, one obvious and unavoidable effect which breaks the isospin symmetry is of course the Coulomb force between protons. But is this the only one? To investigate this question for the $A=54$ mirror pair, the most difficult part is the identification of excited states in the exotic $N=Z-2$ nucleus ${ }^{54} \mathrm{Ni}$. Two different experimental approaches have been successfully employed to study this nucleus: The fusion-evaporation reaction ${ }^{28} \mathrm{Si}\left({ }^{28} \mathrm{Si}, 2 \mathrm{n}\right){ }^{54} \mathrm{Ni}$ and the EUROBALL $\gamma$-ray spectrometer coupled to neutron detectors at the IReS Strasbourg (France) [51] and the fragmentation of a ${ }^{58} \mathrm{Ni}$ beam at relativistic energies at GSI (Darmstadt, Germany) [52]. In these experiments the excitation energies of the first $J^{\pi}=2^{+}, 4^{+}, 6^{+}, 8^{+}$, and $10^{+}$states were measured allowing now for a comparison to the less exotic mirror nucleus ${ }^{54} \mathrm{Fe}$. The quantity $\operatorname{TED}(\mathrm{J})=\mathrm{E}_{J}\left({ }^{54} \mathrm{Ni}\right)+\mathrm{E}_{J}\left({ }^{54} \mathrm{Fe}\right)-2 \mathrm{E}_{J}\left({ }^{54} \mathrm{Co}\right)$, the socalled triplet energy difference, as function of the spin is shown in the lower part of Fig. 9. It is compared to modern shell model calculations which consider both the wellunderstood Coulomb contribution as well as an additional
non-Coulomb isospin-breaking term of isovector origin (for details see Ref. [51]). The result of this comparison is that this nuclear isospin-breaking (NIB) component, although a factor two to three smaller as compared to the Coulomb term, is indeed important and needs to be taken into consideration. Another approach probing isospin mixing at finite temperature is the study of the giant dipole resonance in $N=Z$ nuclei (see Ref. [53]). A detailed discussion of Coulomb energy differences in isobaric multiplets and their interpretation is presented in the review article by Bentley and Lenzi [54].

\subsection{The isoscalar $T=0$ pairing phase}

In the last section we discussed the isospin symmetry, and the effects which break this symmetry, comparing the energies of excited $T=1$ states in an isobaric triplet, i.e. the three isobars with $T_{z}=+1,0$, and -1 . Isospin $T=1$ pairing is dominant at low excitation energies for all known nuclei up to around mass 80 , including those residing along the $N=Z$ line. In these nuclei, proton-proton pairs and neutron-neutron pairs dominate providing properties similar to superfluid helium and superconducting solids. However, there are long-standing theoretical predictions that this situation may change, from a nuclear superfluid dominated by isovector pairing to a structure where isoscalar, i.e. $T=0$, proton-neutron pairing plays a major role, for heavier nuclei towards the exotic doubly-magic nucleus ${ }^{100} \mathrm{Sn}$, the heaviest $N=Z$ nucleus predicted to be bound. Heavy $N=Z$ nuclei with mass $A>90$ are difficult to study since they can only be produced, again employing fusionevaporation reactions, with very low cross-section and in the presence of background from other reaction channels which is many orders of magnitude stronger. The heaviest self-conjugate nucleus, for which excited states have been observed so far, is ${ }^{92} \mathrm{Pd}$. It has been produced as twoneutron evaporation reaction channel of the compound nucleus reaction ${ }^{58} \mathrm{Ni}\left({ }^{36} \mathrm{Ar}, 2 \mathrm{n}\right)$ at GANIL [55] with a relative yield of less than $10^{-5}$ of the total fusion cross-section. Light charged particles and neutrons were detected in coincidence with the emitted $\gamma$ radiation to allow for channel selection. In this experiment three $\gamma$ rays were assigned to the decay of excited states of ${ }^{92} \mathrm{Pd}$ and arranged in a ground-state band based on their relative intensities. The excitation energies of the first $\left(2^{+}\right),\left(4^{+}\right)$, and $\left(6^{+}\right)$ states were then compared to the results of shell model calculations as shown in Fig. 10. Both the experimental and the calculated spectra of ${ }^{92} \mathrm{Pd}$, with four proton holes and four neutron holes relative to the ${ }^{100} \mathrm{Sn}$ core, exhibit a nearly constant energy spacing between consecutive levels. This is in clear contrast to the seniority coupling scheme which dominates for nuclei in the normal isovector pairing phase, for example the $N=50$ isotone ${ }^{96} \mathrm{Pd}$ with four proton holes relative to ${ }^{100} \mathrm{Sn}$. In the latter case, the transition from the ground state to the first excited $2^{+}$state requires the breaking of one $g_{9 / 2}$ proton-hole pair, and therefore the energy spacing between these two levels is rather large. The distance between the subsequent levels then gradually decreases as the angular momentum vec- 


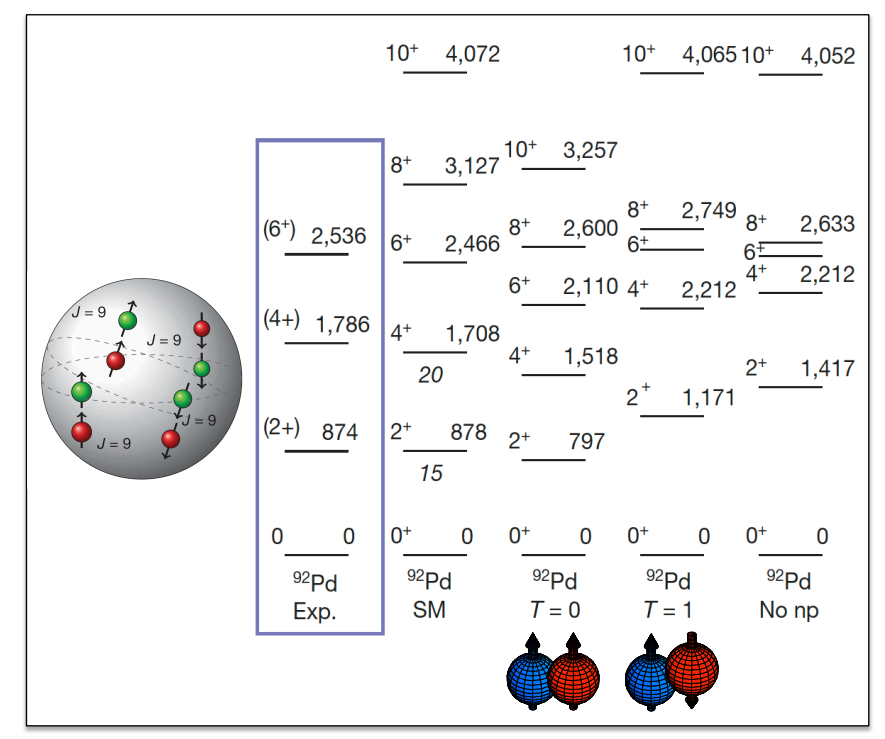

Fig. 10. Left: Comparison between experimental excitation energies in the ground-state band of ${ }^{92} \mathrm{Pd}$ and the results of different shell model calculations. Shell model calculations were performed including either the full proton-neutron pairing (SM), only its $T=0$ component, only its $T=1$ component or no pairing at all (No np). Right: Schematic illustration of the structure of the ground state of ${ }^{92} \mathrm{Pd}$ in the spin-aligned proton-neutron paired phase. Figure adopted from Ref. [55].

tors of the two proton holes align until the maximum spin of $8^{+}$is reached. To examine the influence from different components of the proton-neutron interaction on the excitation spectrum of ${ }^{92} \mathrm{Pd}$, additional shell model calculations were performed including only the $T=0$ component of the pairing, including only the $T=1$ part or finally excluding proton-neutron pairing at all. As shown in Fig. 10, in the latter case a seniority-type spectrum is obtained. For the full calculation (labeled SM in Fig. 10), the calculated excitation energies agree very well with those deduced from experiment. It is evident that the $T=0$ component of the proton-neutron interaction plays an important role for the spectrum of ${ }^{92} \mathrm{Pd}$. This component can be visualized as spin-aligned (deuteron-like) proton-neutron hole pairs with respect to the ${ }^{100} \mathrm{Sn}$ core, spinning around the centre of the nucleus (see Fig. 10). To conclude this section, the structure of the heavy $N=Z$ nucleus ${ }^{92} \mathrm{Pd}$ differs markedly from what has so far been observed in all known atomic nuclei. In this exotic atomic nucleus strongly coupled proton-neutron pairs have a decisive influence on the structure of the nucleus.

\subsection{Shape coexistence in the lead isotopes}

The observation that a particular atomic nucleus can exhibit eigenstates with different shapes appears to be a unique type of behavior in finite many-body quantum systems. States of different shapes are also known to exist in molecules. However, in that case they are based on geometrical arrangements of widely spaced atomic nuclei (constrained by identical chemical bonds). Atomic nuclei do not possess a substructure with widely spaced subunits. The phenomenon of shape coexistence is observed all over the nuclear chart, from the cluster structures observed in light nuclei towards the fission isomers in heavy systems. One prominent example of coexisting spherical and deformed states has been discussed in Section 2.3. There it was reported that the $N=20$ shell closure disappears moving towards the neutron dripline because in ${ }^{32} \mathrm{Mg}$ the deformed two-particle-two-hole configuration $(2 \mathrm{p}-2 \mathrm{~h})$, which comprises the excitation of a neutron pair across the $N=20$ shell gap, becomes energetically favoured as compared to the normal, spherical 0p-0h configuration. Another manifestation of shape coexistence, namely the occurrence of superdeformed bands in nuclei which are spherical in their ground state, will be discussed later in Section 5.1. Here we will briefly mention a very special case of shape coexistence, namely the triple shape coexistence discovered in the heavy neutron-deficient nucleus ${ }^{186} \mathrm{~Pb}$ [56]. Excited states in this nucleus have been populated in the $\alpha$ decay of ${ }^{190} \mathrm{Po}$ which in turn was produced in the fusionevaporation reaction ${ }^{142} \mathrm{Nd}\left({ }^{52} \mathrm{Cr}, 4 \mathrm{n}\right){ }^{190} \mathrm{Po}$ at GSI. At this point, it is worth mentioning again that in such experiments the challenge is to select the nucleus of interest, produced at a very low rate of about 300 atoms per hour, in the presence of a background of other reaction channels which is about a million times higher. In this case the SHIP velocity filter, developed for the studies of superheavy elements (see section 4.1), has been used to separate the ${ }^{190}$ Po nuclei from the unwanted background before their implantation in a position-sensitive Si detector in which the subsequent $\alpha$ decay was detected. The observed fine structure of the $\alpha$ decay allowed for the identification of two excited $0^{+}$states at energies of 532 and $650 \mathrm{keV}$ as shown in Fig. 11. Based on additional experimental information and the energy systematics in the heavier $\mathrm{Pb}$ isotopes (for a detailed discussion see Ref. [56]) it has been possible to assign spherical, oblate and prolate shape to the three $0^{+}$states at 0,532 , and $650 \mathrm{keV}$ excitation energy, respectively. The unique feature of this case of triple shape coexistence is that these three states are the lowest states at all in the energy spectrum of ${ }^{186} \mathrm{~Pb}$. On the theoretical side, the phenomenon of shape coexistence has been studied in the past using a number of different approaches, among them microscopic shell model descriptions and mean-field calculations (for an overview see Ref. [57]). An example of the latter, employing the Gogny force, is shown in Fig. 11 to illustrate the evolution of the potential energy surface for the $A=182-192 \mathrm{~Pb}$ isotopes [58]. These calculations not only nicely describe the experimental excitation energies but furthermore provide explications for the existence of the three low-lying states of different shapes and of the evolution of these minima with the mass number. 


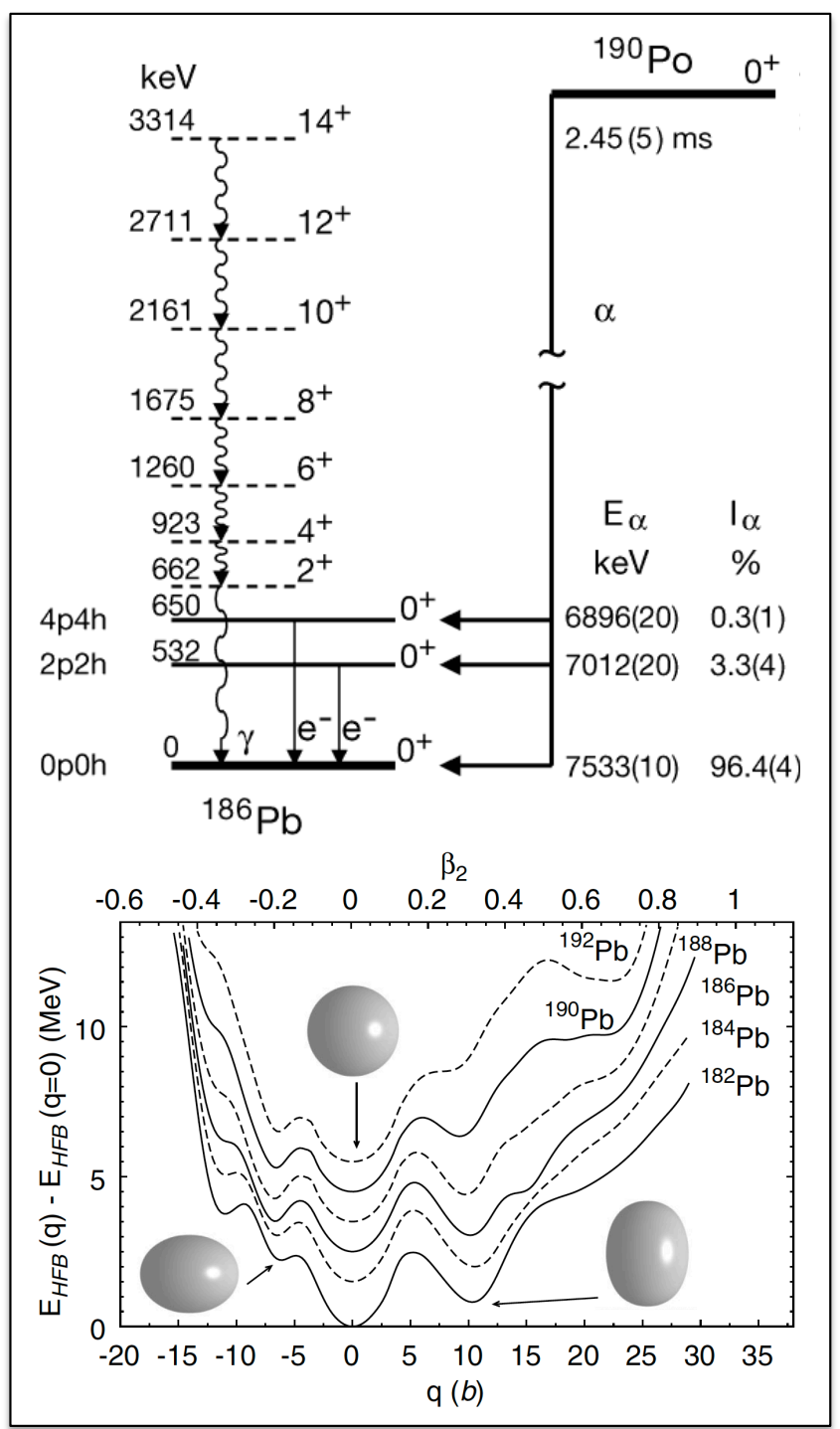

Fig. 11. Top: Scheme of the $\alpha$ decay of ${ }^{190} \mathrm{Po}$ and partial level scheme of ${ }^{186} \mathrm{~Pb}$. Bottom: Calculated potential energies for the $\mathrm{Pb}$ isotopes as function of the deformation. Figure adopted from Refs. [56, 58].

\section{The heavy end of the nuclear chart}

\subsection{The synthesis of superheavy elements (SHE)}

Until the beginning of the 1950s it was not expected that elements beyond Fermium $(Z=100)$ exist. At that time it was still assumed that an atomic nucleus consisting of nucleons can be regarded in analogy to a liquid drop built out of atoms. This liquid drop model allowed to describe many at that time modern phenomena like neutron-induced fission and neutron capture. In this model, it was expected that around proton number 100 the repulsive Coulomb force would win over the attractive nuclear surface tension and the nuclei would decay spontaneously. As mentioned in the Introduction, only around 1950 it was realized that many nuclear properties are not smooth, uniform functions of the proton and neutron numbers, but show pronounced non-uniformities for certain magic proton and neutron numbers. Nuclei consisting of a magic number of protons or neutrons have increased binding energies relative to the average trend. The same effect is known from atoms (remember the increased stability of the nobel gases) and in analogy to atomic physics the nuclear shell model was developed. By the end of the 1960s, it had been concluded that these shell effects are the reason why atomic nuclei can be more stable than expected from the liquid drop model and therefore nuclei beyond Fermium can exist. Early calculations predicted the nucleus with $Z=114$ and $N=184$ to be the centre of an island of long-lived superheavy nuclei far beyond the limit of stability predicted by the liquid drop model [59-61]. This result stayed practically unchallenged until the late 1990s, when more refined models, based on realistic effective nucleonnucleon interactions, were applied to superheavy nuclei. However, the microscopic models are still uncertain when extrapolating in $Z$ and the mass number $A$ leading to the fact that there is no consensus among theorists with regard to what should be the next doubly magic nucleus beyond ${ }^{208} \mathrm{~Pb}(Z=82, N=126)$ [62]. It is furthermore expected that superheavy elements can exist in a variety of shapes, including spherical, axial and triaxial configurations. As a consequence, besides the spherical shell closures mentioned above, also deformed shell gaps are anticipated. A sketch of the situation as predicted by theoretical calculations is shown in Fig. 12.

On the experimental side, our present knowledge about the heavy mass end of the nuclear chart beyond the heaviest radioactive element existing in nature, Uranium with 92 protons, has been accumulated in three clearly separated phases during the last century. Following a suggestion by Enrico Fermi in 1934 (only two years after the discovery of the neutron by Chadwick!) the eight transuranium elements from Neptunium $(Z=93)$ up to Fermium $(Z=100)$ have been synthesized by neutron capture and

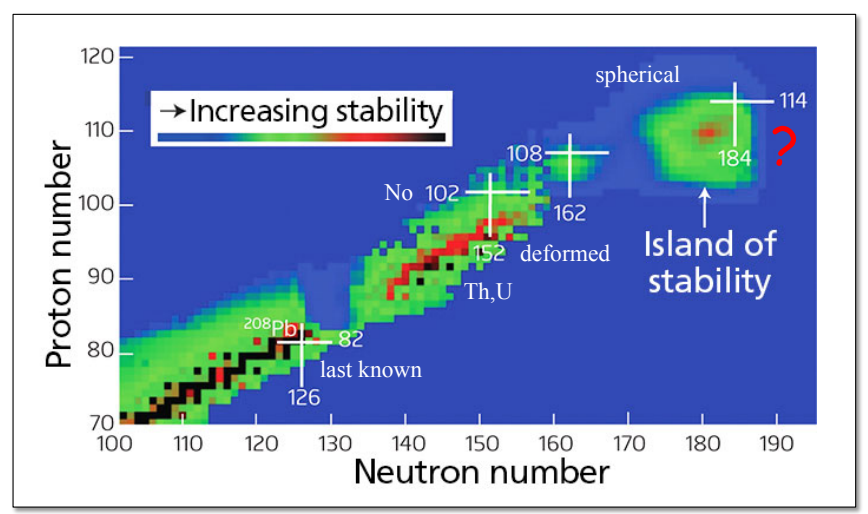

Fig. 12. Sketch of the heavy end of the nuclear landscape. Besides the heaviest known doubly-magic nucleus ${ }^{208} \mathrm{~Pb}$, theoretically predicted spherical and deformed shell gaps are indicated by white lines. The region around the predicted spherical doubly-magic nucleus ${ }^{298} 114$ is called island of stability. Between the main continent and this island of stability a region of deformed nuclear shapes is predicted. 


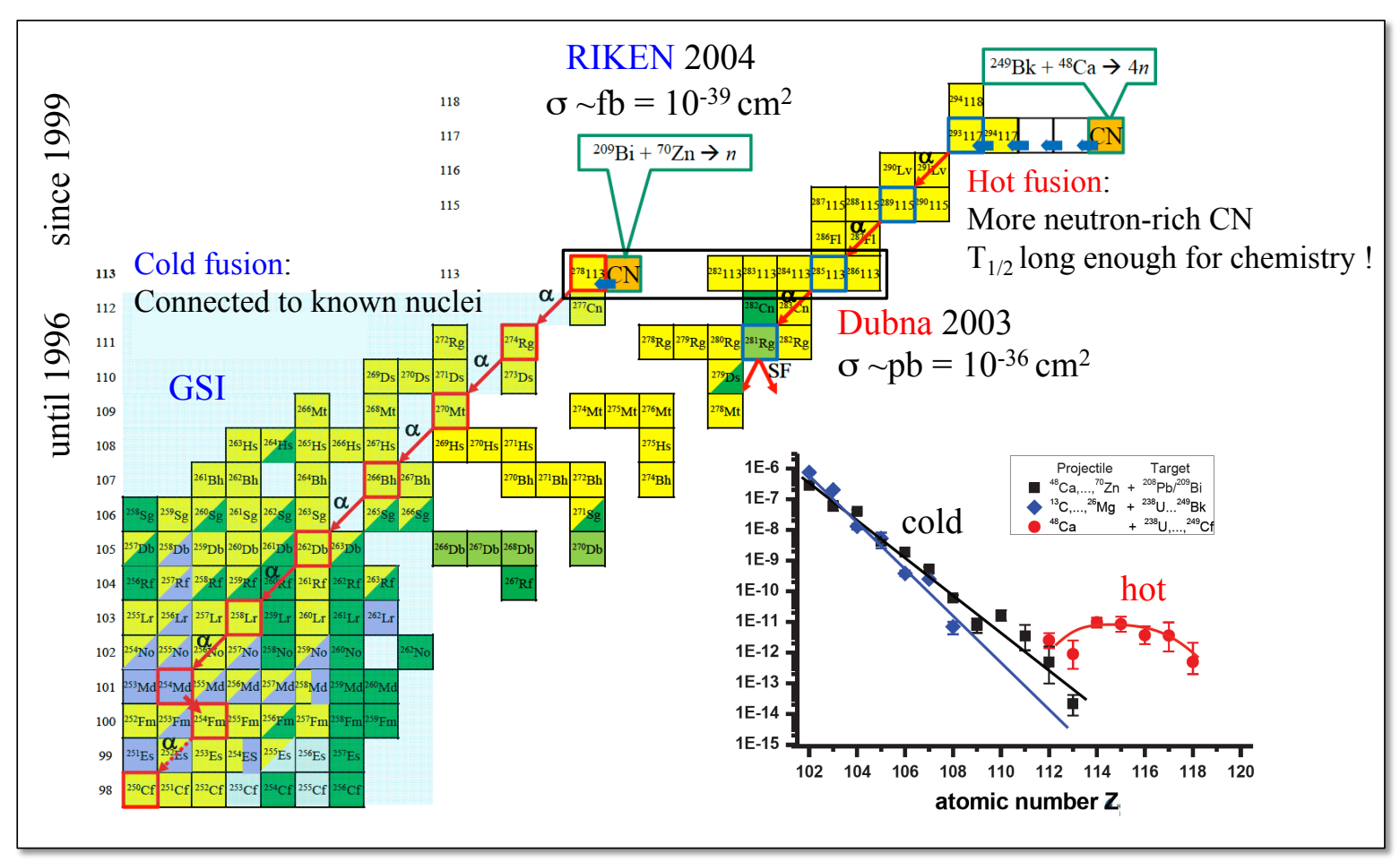

Fig. 13. Summary of the current experimental knowledge of the upper end of the nuclear chart. The elements in the range $Z=107-112$ were discovered using cold fusion reactions at GSI, while the heavier elements up to element 118 were produced in hot fusion reactions at Dubna. Note that element 113 is the only SHE which was synthesized using both cold and hot fusion reactions (see text for details). The inset shows experimental cross sections for cold and hot fusion reactions as a function of the atomic number $Z$ of the synthesized element. Figure adopted from Refs. [63,64].

successive beta-decay in the years 1940-1955. It is interesting to remember that it was this idea by Fermi which lead to the discovery of the nuclear fission in 1938. However, this path to heavier nuclei stops at Fermium, since none of its isotopes decays via $\beta$ decay due to the short $\alpha$ decay and fission half-lives. The elements with $Z=101-106$ were then produced between 1955 and 1974 by bombarding the heaviest elements available, namely the transuranium elements produced in a high-flux reactor, with light ions. The typical cross sections for such fusion-evaporation reactions are much smaller than the ones for neutron capture used for the lighter heavy elements making the experiments much more difficult. Note that at that time, only the University of Berkeley in the US and the russian research center at Dubna were able to perform SHE research since only the nuclear weapon nations were in the possession of installations for the production of large samples of the heavy actinides, which were needed as target material. A reminder of this cold war period is the tough fight about the names of the new elements with proton number $Z=101-106$.

With each new element, the production cross section decreases by a factor of three or four (see inset of Fig. 13) and also the half-live drops drastically. It was therefore evident that in order to proceed to even heavier systems new reactions as well as new identification methods had to be searched for. Concerning the first, it had been realized that instead of bombarding heavy actinides with light ions, the fusion between a doubly-magic $\mathrm{Pb}$ target with heavy ions of rather low energy below the Coulomb barrier leads to a much suppressed fission probability and therefore higher yields. In this so-called cold-fusion reaction, the excitation energy of the compound nucleus amounts to only about $10-20 \mathrm{MeV}$ and already the evaporation of one (or two) neutrons cools it down to a state stable against fission. However, only in one out of thousand cases the compound nucleus gets rid of its excitation energy before fission occurs. Lead and Bismut are best suited as target material because both spherical nuclei are stabilized by the $Z=82$ and $N=126$ shell closures. As beams neutron rich isotopes close to shell closures are best. For the identification of the new very short-lived elements, a fast physical technique has been applied instead of the slow chemistry. The trick is to separate the reaction products from the beam using a velocity filter, to implant them into a Si detector and finally to identify them via their $\alpha$ decay. To synthesize the elements with $Z=107-112$ this correlation technique and cold fusion reactions have been employed using the velocity filter SHIP at GSI. An ion beam with a very high intensity of about $3 \times 10^{12}$ ions/sec hits a target wheel which rotates synchronously with the pulse structure of the beam in order to distribute the heat over a larger area. Nearly all ions pass through the target without interaction. Only about once a week, a fusion product is produced, which continues to move in the same direction as the beam ions, however with a reduced velocity. The electric and mag- 
netic fields of the velocity filter SHIP are chosen such that the fusion products hit a $\mathrm{Si}$ detector whereas the ion beam is directed onto a beam catcher. In the position sensitive Si detector, position, time and deposited energy of the implants are registered as well as time and energy of the subsequent $\alpha$-decays. Using this technique elements up to $Z=112$ could successfully be identified until 1996 , produced with cross sections as low as $10^{-36} \mathrm{~cm}^{2}(\mathrm{pb})$.

Around $Z=112$ the applicability of the cold fusion reactions comes to an end. The main reason is that the cross section for the production of the evaporation residue decreases exponentially with the increase of the proton number of the compound nucleus because of the increase of the Coulomb repulsion force. Note that when the compound nucleus $Z$ changes from 102 to 113, the cross section drops by a factor of $10^{8}$ ! Furthermore the evaporation residue produced in cold fusion reactions are some 10-15 mass units shifted from the $\beta$-stability line which leads to a considerable decrease in their half-lives further hindering its detection. At that point it was clear that one had to look for other reactions when aiming for the synthesis of even heavier elements. In order to decrease the factors hindering fusion it was suggested that the use of neutronrich isotopes of the actinides as targets and the doubly magic nucleus ${ }^{48} \mathrm{Ca}$ would be a good choice. For example, in the reaction ${ }^{244} \mathrm{Pu}+{ }^{48} \mathrm{Ca}\left(Z_{P} \cdot Z_{T}=1880\right)$, the Coulomb repulsion decreases by almost $40 \%$ as compared to the cold fusion reaction ${ }^{208} \mathrm{~Pb}+{ }^{76} \mathrm{Ge}\left(Z_{P} \cdot Z_{T}=2624\right)$. This drop is expected to reduce the factors hindering fusion in these reaction, which are called hot fusion reactions because the compound nucleus is produced at significantly higher excitation energies of 30 to $55 \mathrm{MeV}$ leading to the evaporation of a larger number of neutrons as compared to cold fusion reactions. Another feature of these proposed hot fusion reactions is that they produce more neutronrich compound nuclei. For example, the compound nucleus ${ }^{292} 114$, produced via the hot fusion reaction ${ }^{244} \mathrm{Pu}+{ }^{48} \mathrm{Ca}$, has 8 neutrons more as compared to the one formed in the cold fusion reaction ${ }^{208} \mathrm{~Pb}+{ }^{76} \mathrm{Ge}$ reaction. Consequently, longer half-lives of the evaporation residue can be expected.

In 1999 , the ${ }^{244} \mathrm{Pu}+{ }^{48} \mathrm{Ca}$ reaction was successfully used to synthesize the element 114 using the gas-filled recoil separator DGFRS at the Joint Institute for Nuclear Research (Dubna, Russia). This experiment opened a new era in SHE research which lead to the discovery of all elements up to $Z=118$ using similar hot fusion reactions in the following years. The current experimental information about SHE is summarized in Fig. 13. It was found that the cross sections for hot fusion reactions are indeed relatively large, in fact all elements up to 118 can be produced in this way with a larger cross section as compared to the one of the cold fusion reaction used to synthesize element 112 (see inset of Fig. 13). Besides the cross section there are two major differences between the production of SHE using cold and hot fusion reactions: First the isotopes produced in hot fusion reactions do not decay into the region of known isotopes (see Fig. 13). Therefore the assignment must always be based on indirect arguments in this case. The second difference concerns the half-life of the produced isotopes. Due to their larger neutron excess the isotopes produced in hot fusion reactions have longer half-lives which in some cases are even long enough to perform chemistry. There is one element, namely element 113, which has been synthesized both in cold and hot fusion reactions. This case nicely illustrates the complementary of the two techniques. At RIKEN the isotope ${ }_{278} 113$ was populated as one-neutron evaporation channel in the reaction ${ }^{209} \mathrm{Bi}+{ }^{70} \mathrm{Zn}[65]$. The cross section for this reaction is extremely low, at the very limit of experimental feasibility, as evidenced by the fact that within 553 days with beam on target the decays of only three ${ }^{278} 113$ nuclei have been observed! Most important, in one of the events a long chain of six $\alpha$ particles was observed which linked the new isotope to well-known and previously studied nuclei. The half-life of this isotopes is very short, below one millisecond. In Dubna, on the other hand, the hot fusion reaction ${ }^{243} \mathrm{Am}+{ }^{48} \mathrm{Ca}$ was used to produce ${ }^{288} 115$ and as its $\alpha$-decay daughter ${ }^{284} 113$, a more neutron-rich isotope of element 113, with much higher cross section [66]. However, in this case each of the isotopes in the decay chain was new and had never been studied before. It is therefore necessary to study the chemical properties of the final atom in the chain to prove that it was Dubnium $(Z=105)$. The decision which of the two experiments, i.e. less ambiguity in the identification vs. larger number of events, will be given preference when it comes to the assignment of the naming rights is expected with great suspense.

Considering all information about superheavy elements available today, i.e. fission and $\alpha$ decay half-lives and decay modes, one remarkable observation is that nearly all isotopes above Rutherfordium $(Z=104)$ are $\alpha$ emitters. That means that they are more stable against fission than the isotopes of Rutherfordium and Nobelium. Calculations have shown that this stability comes from the ability of the nucleus to deform, with not only quadrupole but also higher multipole moments. Indeed, deformed shell gaps at neutron numbers $N=152$ and $N=162$ have been proposed (see Fig. 12) which in the first case have already been confirmed experimentally in recent mass measurements of No isotopes [67] and also by $\gamma$-ray spectroscopy as will be discussed in the next section.

We close this section with the remark that the next heavier, so far undiscovered elements 119 and 120 have already been searched for in a number of experiments performed at different laboratories in recent years. However, the results of these activities have not yet been presented. For more detailed information about the exciting past and presence of SHE research the reader is referred to Refs. [63, 64] and references therein.

\subsection{The spectroscopy of transfermium nuclei}

Nuclear spectroscopy is a very powerful tool to give access to nuclear structure and the properties at excitation energies up to several MeV. Spectroscopic techniques can probe the single-particle states around the Fermi level 
and thus help to improve predictions of nuclear properties in nuclei not accessible for experimental studies today. Due to the very low production cross sections, until now it has not been possible to study SHE by means of in-beam spectroscopic techniques. On the other hand, many of the transfermium nuclei $(Z>100)$ are produced in fusion-evaporation reactions with cross-sections large enough, i.e. in the nb to $\mu \mathrm{b}$ range (see Fig. 13), to allow for their study using both in-beam and decay spectroscopy. Detailed spectroscopic studies of nuclei from $\mathrm{Cm}$ to $\mathrm{Db}$ allow to pin down the evolution of single-particle states in this region and provide valuable information on the details of the shell correction in heavy nuclei. Ultimately, such data can help to define the extent and location of the SHE island of stability.

As discussed in the last section, the stability of the isotopes beyond Fermium is predicted to arise from nuclear deformation effects. The first direct proof of this prediction was presented by Reiter et al. in 1999, who observed a rotational band built on the ground state of ${ }^{254}$ No $(Z=102)$ [68]. Excited states of this nucleus were populated in the reaction ${ }^{208} \mathrm{~Pb}\left({ }^{48} \mathrm{Ca}, 2 \mathrm{n}\right)$ with a cross section of $3 \mu \mathrm{b}$ in an experiment performed at the Argonne National Laboratory (USA). The challenge was to identify $\gamma$ rays emitted from excited states of ${ }^{254}$ No in a background of more than 10000-times more intense fission $\gamma$ rays. This was accomplished by combining efficient $\gamma$-ray detection power with the ability of a residue separator to identify unambiguously very weekly produced evaporation residues. Prompt $\gamma$ rays were detected using the Gammasphere spectrometer (see next section) positioned around the target wheel. The evaporation residues were separated from the beam by a fragment mass analyzer (FMA). At the focal plane of the FMA, the particles were detected in a position-sensitive Si detector. The production of ${ }^{254} \mathrm{No}$ was unambiguously demonstrated by the observation of subsequent $\alpha$ decays at the same position in the Si detector where the implant has been detected. Both the $\alpha$ energies as well as the decay time correspond to the ones known for the decay of ${ }^{254}$ No. The $\gamma$ rays, which where detected in coincidence with the ${ }^{254}$ No residues, form a regular rotational band which in subsequent experiments was further extended up to spin $24^{+}[69]$ as shown in Fig. 14 Besides the first direct determination of the ground state deformation of a transfermium isotope, the observation of the rotational band up to spin $14^{+}$in the pioneering work of Ref. [68] had another important implication. It demonstrated that neutron evaporation can compete up to at least this spin against fission. Hence, a fission barrier must still exist up to this angular momentum - a fact, which is not at all obvious for a nucleus which exists only due to shell effects.

In the following years rotational bands were studied in a number of additional even and odd nuclei in this region, produced with cross sections as low as $17 \pm 3 \mathrm{nb}$ in the case of ${ }^{256} \operatorname{Rf}(Z=104)$ [70]. The systematic study of rotational properties such as the kinematic and dynamical moments of inertia revealed subtle effects believed to be related to changes in alignment and pairing cor-

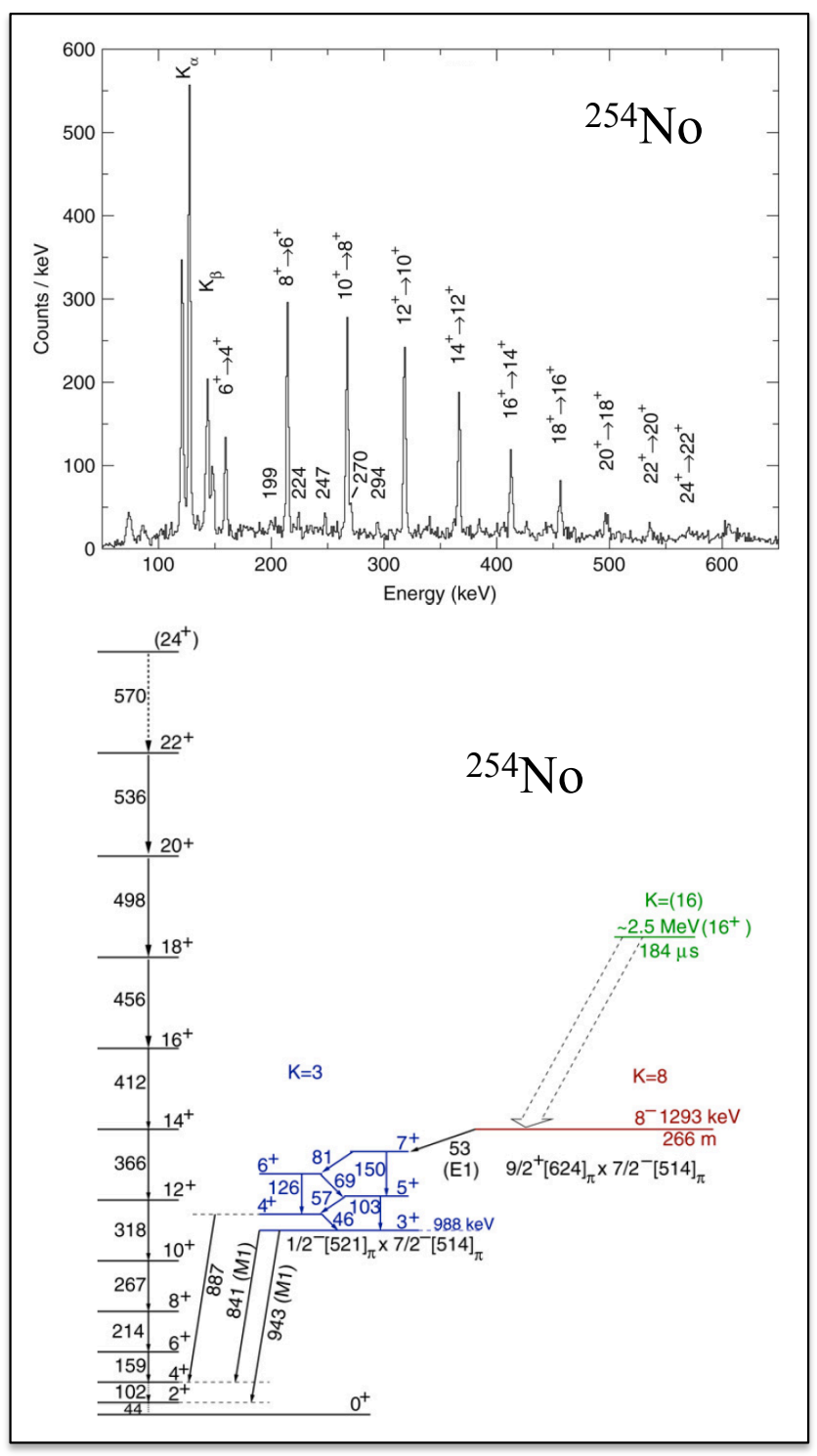

Fig. 14. Top: $\gamma$-ray spectrum of ${ }^{254} \mathrm{No}$, showing the rotational ground state band up to a spin of $24 \hbar$. Bottom: Level scheme of ${ }^{254}$ No showing the rotational band on the left and three excited structures including two isomeric states on the right. Adopted from Refs. [69,72].

relations [69]. Additional valuable information can be obtained from the study of excited states of non-collective character. Since the nuclei in this region are deformed, the degenerate spherical single-particle orbitals split in a well defined manner into components according to the projection of the angular momentum onto the symmetry axis of the nucleus as first described by Nilsson in 1955 [71]. Orbitals which are relevant for the existence or not of spherical shell gaps dive down and come close to the Fermi level in lighter deformed nuclei and thus play a key role in the formation of excited states. In Ref. [72] three excited structures were identified in ${ }^{254} \mathrm{No}$ (see Fig. 14), two of which with long half-lives in the $\mu \mathrm{s}$ and ms range, respectively. Two of them correspond to configurations in which a pair 
of protons is broken and the two unpaired protons occupy different single-particle orbitals. The third most probably involves both a broken proton and a broken neutron pair. This new experimental data allowed the position of a particular Nilsson orbital to be determined, which is of special interest as the level originates from the spherical $2 f_{5 / 2}$ orbital from above the possible spherical $Z=114$ shell gap. The separation and position of the $2 f_{7 / 2}-2 f_{5 / 2}$ spin-orbit partners plays a crucial role for predictions of the location of the island of stability.

An overview of recent in-beam and decay spectroscopy of transfermium nuclei is given in Ref. [69], for a more general glance the reader is referred to Ref. [73].

\section{Atomic nuclei under extreme conditions}

The study of the nucleus under extreme conditions with respect to angular momentum and excitation energy played an important role in nuclear structure physics during the last decades. Deformed nuclei can rotate with high angular frequencies (of the order of $10^{21} \mathrm{~Hz}$ for example for a mass $A \sim 70$ nucleus at spin $70 \hbar$ ) which implies enormous Coriolis forces. On the other hand, a nucleus can stand around $5 \mathrm{MeV}$ excitation energy, which corresponds to a maximum temperature of about $5.8 \times 10^{10} \mathrm{~K}$. The nuclear level density increases exponentially with the excitation energy which allows to apply a statistical treatment and the concept of nuclear temperature. The question to answer is how tens or even hundreds of strongly interacting nucleons react when they are exposed to the mentioned extreme conditions.

In the first decades of experimental nuclear structure physics, experimental studies were limited to the investigation of the properties close to the ground state. However, in the same way as the availability of radioactive ion beams allowed during the last decades to study regions of the nuclear landscape further away from the valley of stability (see first part of this article), other technical advances, namely stable heavy ion beams and efficient $\gamma$-ray spectrometer, facilitated the exploration of the excitation energy versus spin plane, i.e. the systematic study of the nuclear properties as a function of excitation energy (temperature) and angular momentum (rotational frequency).

The origin of the so-called high spin physics dates back to the 1970s. While already in 1963 a regular rotational band, namely the ground state band of ${ }^{160} \mathrm{Dy}$, was observed for the first time up to the $10^{+}$state [74], only in 1972 Johnson and collaborators discovered irregular spacings within this band in the spin range $14^{+}-18^{+}[75]$. In both experiments the $\alpha$-induced fusion-evaporation reaction ${ }^{160} \operatorname{Gd}(\alpha, 4 \mathrm{n}){ }^{160}$ Dy was employed but while in the first the emitted $\gamma$ radiation was detected in a $\mathrm{NaI}$ scintillator, in the second a $\mathrm{Ge}(\mathrm{Li})$ semiconductor detector was used providing a much better energy resolution. Visualizing the rotational band in a moment of inertia vs. rotational frequency diagram as it is shown in Fig. 15 the irregularity appears as a backbending, the frequency of the collective rotation decreases while the moment of inertia (and the

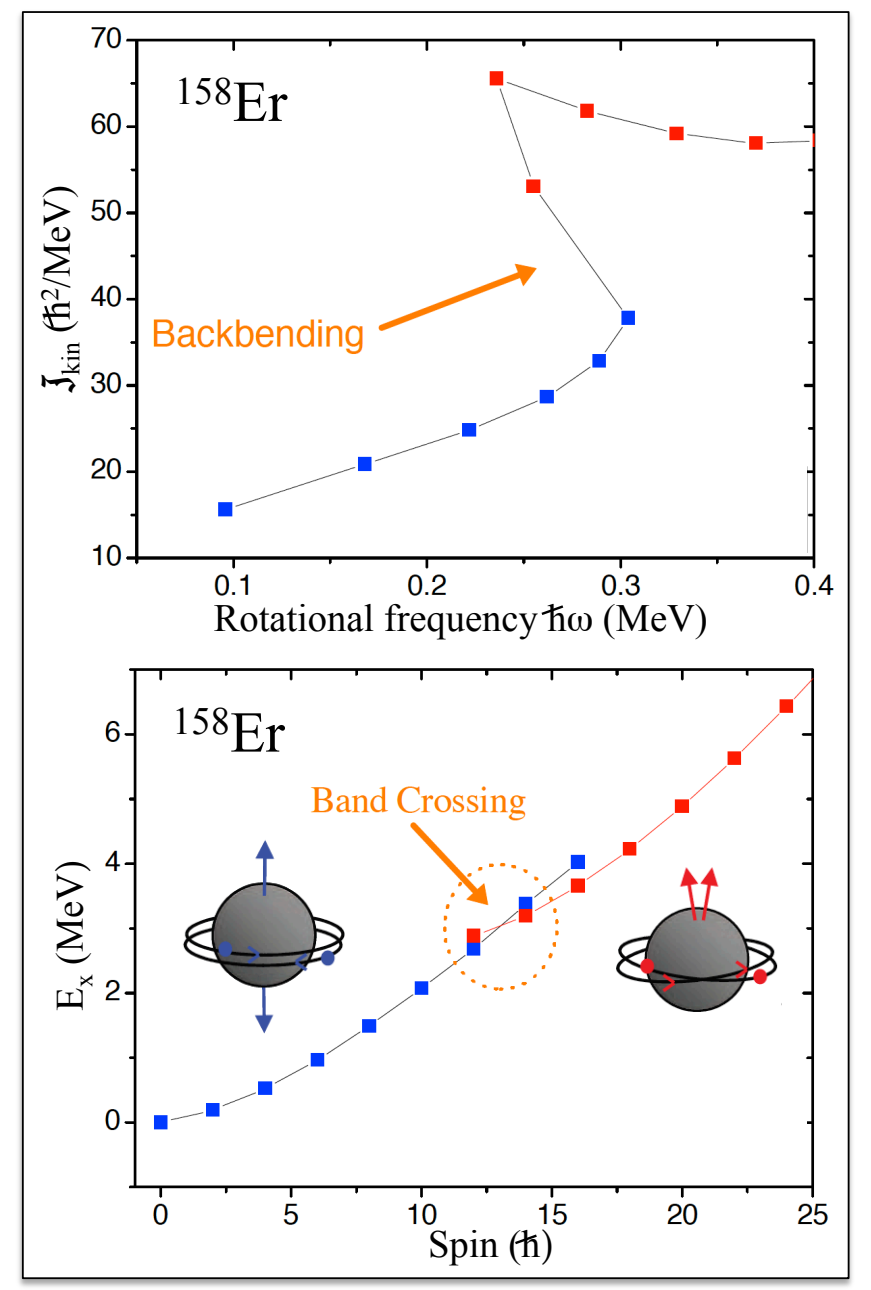

Fig. 15. Top: Kinematical moment of inertia as a function of the rotational frequency for the yrast sequence of ${ }^{158} \mathrm{Er}$. The S-shape is usually referred to as a backbending and occurs due to the alignment of a pair of $i_{13 / 2}$ neutrons. Below this critical frequency value, the yrast sequence is composed of the ground-state band, but after the backbend the $i_{13 / 2}^{2}$ configuration becomes yrast. Bottom: Excitation energy versus spin diagram for the yrast sequence of ${ }^{158} \mathrm{Er}$. In this presentation the backbending appears as a crossing between two bands, namely the ground state band and the band built on an aligned $i_{13 / 2}$ neutron pair, around spin $12^{+}-14^{+}$.

total spin) continues to increase. Choosing instead a presentation in an excitation energy vs. spin diagram, the same irregularity shows up in form of a crossing between two rotational bands with different moments of inertia (see Fig. 15). The expressions backbending and band crossing indeed describe the same phenomenon, namely the fact that at a certain rotational frequency, a configuration different from the ground state configuration may become energetically favoured.

Also in other nuclei in the rare earth region similar features were observed [76]. Soon after the experiment, this observation has been interpreted by Stephens and Simon as being due to the breaking of one pair of neutrons in the 
high- $j$ intruder orbital $i_{13 / 2}$ [77]. In contrast to this picture, Mottelson and Valatin predicted already in 1960s a coherent collapse of pairing correlations at a certain critical rotational frequency, corresponding to a phase transition from the superfluid to a non-superfluid state [78]. The validation of the explanation by Stephens and Simon came in 1977 from the observation of a second irregularity in the ground state rotational band of ${ }^{158} \mathrm{Er}$ in the region around spin $32^{+}$, corresponding to a second pair of nucleons, this time protons in the $h_{11 / 2}$ orbital [79]. Note that only the availability of first heavy-ion accelerators at several laboratories in the 1970s allowed for the observation of rotational bands up to spins as high as $32^{+}$, in the case of ${ }^{158} \mathrm{Er}$ populated in the reaction ${ }^{122} \mathrm{Sn}\left({ }^{40} \mathrm{Ar}, 4 \mathrm{n}\right){ }^{158} \mathrm{Er}$.

\subsection{Multi-detector $\gamma$-ray spectrometer and the discovery of superdeformation}

The discovery of the backbending phenomenon in several rare earth nuclei and the systematic study of a number of rotational bands up to intermediate spin in other mass regions evidenced the importance of $\gamma \gamma$ coincidence information and consequently in the 1980s arrays consisting of 5 to 20 individual Ge detectors were put together replacing the two $\mathrm{Ge}(\mathrm{Li})$ detectors which have been the standard setup in $\gamma$-ray spectroscopy for many years. Around the same time first escape-suppression shields were introduced. In $\mathrm{Ge}$, the cross-section for Compton scattering dominates for $\gamma$-ray energies above $180 \mathrm{keV}$ and the probability that the Compton-scattered $\gamma$ ray escapes from the Ge detector is high. Therefore, the peak-to-Compton ratio of the first Ge detectors was poor, with more than $90 \%$ of the interactions contributing to the Compton distribution and only less than $10 \%$ to the full-energy peak. The Compton continua of all the $\gamma$ rays summed and made it difficult to detect a weak full-energy peak on the large Compton background created by the most intense $\gamma$ rays. Surrounding the Ge crystal by a scintillator, initially in most cases NaI, the majority of the $\gamma$ rays that are scattered out of the Ge detector are detected in this scintillator producing a veto signal which allows to reject the partly absorbed events before the $\gamma$-ray spectrum of the Ge detector is produced. In this way the background in this spectrum could be drastically reduced thus increasing the level of sensitivity.

Based on this technical advancement the first discrete superdeformed (SD) rotational band has been observed by Twin et al. in 1986 in the nucleus ${ }^{152} \mathrm{Dy}$ [80]. This nucleus was produced at high angular momentum at the Daresbury Laboratory (UK) using the heavy-ion induced fusion-evaporation reaction ${ }^{108} \mathrm{Pd}\left({ }^{48} \mathrm{Ca}, 4 \mathrm{n}\right){ }^{152} \mathrm{Dy}$. The $\gamma$ radiation emitted in the decay of the excited states of this nucleus was detected by the TESSA3 spectrometer which consisted at the time of the experiment of twelve escapesuppressed Ge detectors. The efficiency of this instrument was high enough to allow the observation of an extremely regular rotational band which collects only about $1 \%$ of the total intensity of the main reaction channel ${ }^{152} \mathrm{Dy}$. The $\gamma$-ray spectrum of this band is shown in Fig. 16 . The nearly constant spacing of $47 \mathrm{keV}$ between neighbouring lines indicates a very high moment of inertia corresponding to a strongly deformed shape of the nucleus. The existence of second minima in the nuclear potential corresponding to very elongated or superdeformed shapes had been predicted since long time. The single-particle energies in a nucleus depend on the shape of the potential. For spherical shape, large energy gaps occur for the "magic numbers" of nucleons. However, adding more and more nucleons outside closed shells leads to deviations from sphericity and energy gaps occur for different nucleon numbers at different deformations. The total energy which, neglecting residual interactions, is the sum of the single-particle energies of all protons and neutrons in the nucleus under study, is a function of the deformation parameter and can have more than one minimum. Excited states in this sec-

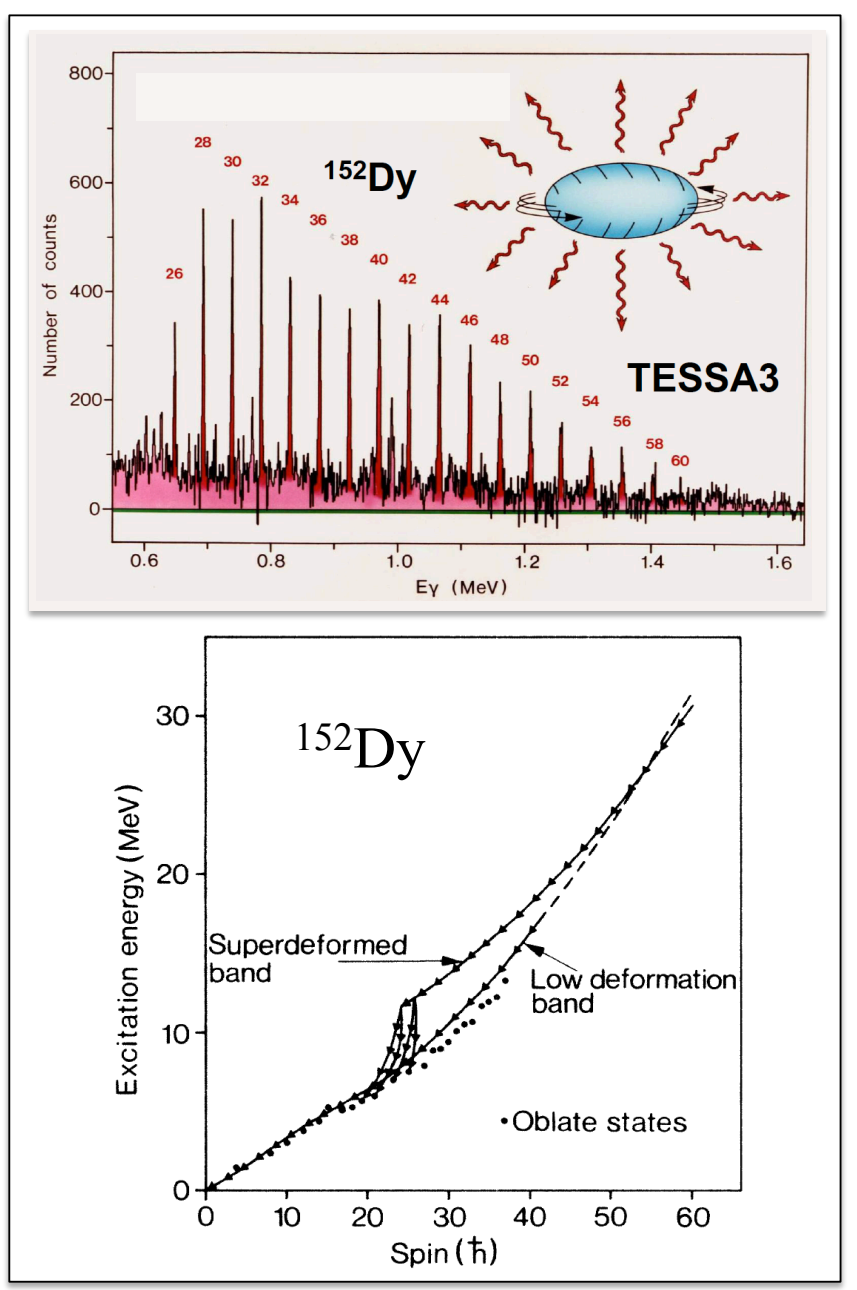

Fig. 16. Top: $\gamma$-ray spectrum of the superdeformed band in ${ }^{152}$ Dy discovered with the TESSA3 spectrometer at the Daresbury laboratory. Note the nearly constant spacing of $47 \mathrm{keV}$ between two successive lines. Bottom: Non-collective states and deformed bands in ${ }^{152}$ Dy in the excitation energy versus spin plane. Some typical decay paths deexciting the superdeformed band to the oblate states are illustrated. Figure adopted from Ref. [80]. 
ond minimum were first observed in an indirect way as resonances in the fission cross sections of nuclei in the actinide region in the 1960s. However, the direct observation of the $\gamma$ rays emitted in the decay of the superdeformed states only became possible with the increased sensitivity of the $\gamma$-ray spectrometer available in the 1980s. As illustrated in Fig. 16, at low excitation energy ${ }^{152}$ Dy has a slightly oblate shape $(\beta \sim-0.1)$ and its yrast line is formed by irregularly spaced single-particle type excitations. At intermediate spin, a deformed rotational band is observed corresponding to a prolate deformation of about $\beta \sim 0.1$. It is only at very high angular momentum that the superdeformed band becomes energetically favoured because of its large moment of inertia. One of the main questions since the first observation of a superdeformed band was how such a SD band decays into the normal deformed or spherical minimum. The observation of this decay, which is schematically indicated in Fig. 16, is hindered by several facts: At excitation energies of several $\mathrm{MeV}$ corresponding to the second minimum the density of states in the first minimum is very high which leads to a fragmentation of the decay paths and as a consequence to low intensities of the individual $\gamma$ transitions which are furthermore of relatively high energy so that the detection efficiency of the Ge detectors is low. In addition the very different structure of the states in the two minima implies low transition probabilities of the transitions between them. Considering furthermore the weak population of the superdeformed structures in fusion-evaporation reactions (because they are yrast only at very high spin) it is not surprising that it was not possible to observe the decay out of a superdeformed band with the first generation of $\gamma$-ray spectrometer such as TESSA or NORDBALL. Fortunately, the history of high-spin physics did not stop at this point. On the contrary, the discovery of superdeformation in 1986, which was considered as one of the major physics discoveries at that time [81], served as starting shot for the development of even larger and therefore more efficient $4 \pi \gamma$-ray spectrometer thus heralding a new era of the investigation of the behaviour of atomic nuclei at high excitation energy and angular momentum.

\section{$5.24 \pi \gamma$-ray spectrometer and the boom of high-spin physics}

The first generation $\gamma$-ray spectrometer extended considerably the region of the excitation energy versus angular momentum plane accessible for experimental studies. For the first time, it became possible to study the behaviour of atomic nuclei at excitation energies up to 30 $\mathrm{MeV}$ and in the spin range up to $60 \hbar$. In light of this new perspective two large-scale projects were started at the end of the 1980s aiming for the construction of arrays of escape-suppressed Ge detectors in $4 \pi$ geometry with a photopeak efficiency of around $10 \%$. To cope with the cost of such arrays, large collaborations were formed aiming for the construction of Gammasphere in the US and EUROBALL in Europe. Although using different technical approaches and geometries(e.g. Gammasphere consists of 110 individual Ge crystals while EUROBALL comprises a total of 239, many of them mounted in composite detectors) both projects reached their goal and both instruments started operation in the 1990s. A detailed description of these projects is presented in the review article by Eberth and Simpson [82]. The experiments performed with Gammasphere at the Argonne and Lawrence Berkeley National Laboratories and EUROBALL at the LNL Legnaro (Italy) and the IReS Strasbourg (France) led to a tremendous gain in the knowledge of the nuclear structure at high excitation energy and angular momentum.

As hoped for with the improved efficiency of these new instruments the first discrete linking transitions which connect SD and normal yrast states in one step were finally observed in the nucleus ${ }^{194} \mathrm{Hg}$ in 1996 . Note that at that time more than $100 \mathrm{SD}$ bands in the mass 150 and 190 regions had been found, but for none of these bands the exact excitation energies, spins and parity could be determined. In the case of ${ }^{194} \mathrm{Hg}$ [83], studied with Gammasphere at the LBNL employing the reaction ${ }^{150} \mathrm{Nd}\left({ }^{48} \mathrm{Ca}, 4 \mathrm{n}\right)$, four high-energetic (3.5-4.5 MeV) electric dipole one-step linking transitions carrying about $5 \%$ of the SD band intensity have been observed and thereby energy and spin of the SD band fixed. The SD band is observed in the spin range from $10 \hbar$ to $50 \hbar$ and at the bottom of the band,

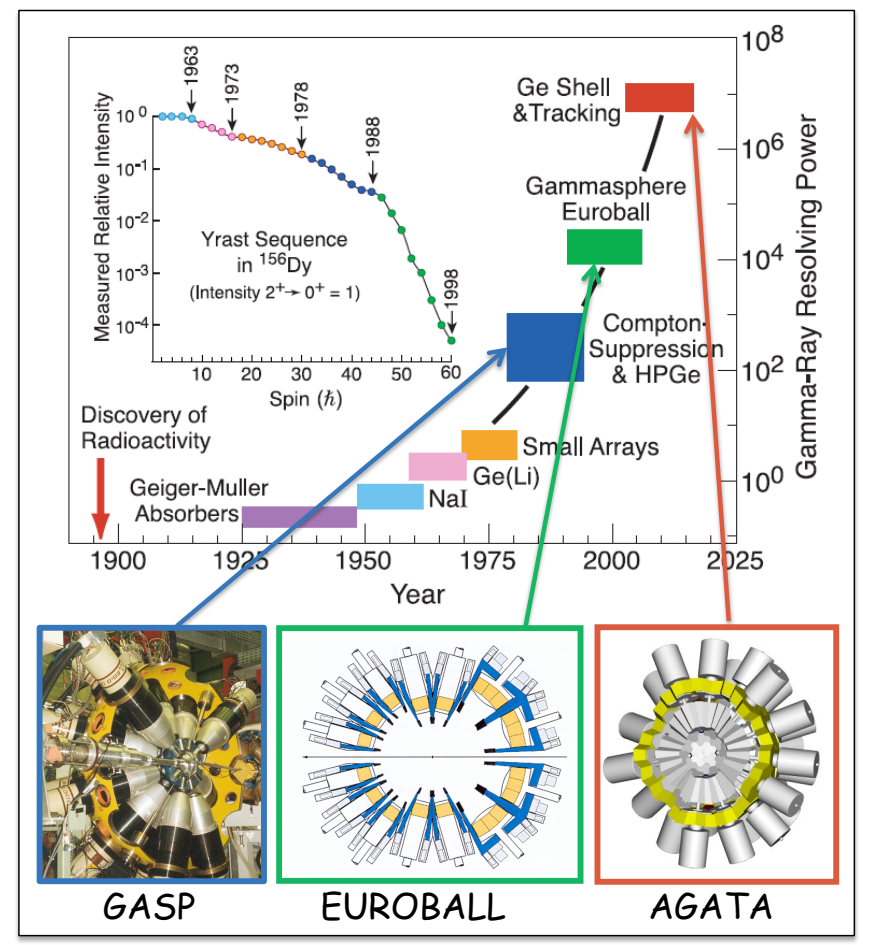

Fig. 17. Overview of the sensitivities reached with different past and present $\gamma$-ray spectrometer. The state-of-the-art instruments EUROBALL and Gammasphere, operational since the mid-ninetees, allow to study excited states down to intensities of about $10^{-6}$ of the production cross section. The next generation of $4 \pi$ tracking arrays (AGATA, GRETA) will have even higher sensitivities. 
where the decay-out occurs, the SD states lie about 4.2 $\mathrm{MeV}$ above the ND yrast levels of the same spin. In 2002, sixteen years after its discovery, also the lowest SD band in ${ }^{152}$ Dy was connected to the remaining level scheme via the observation of four weak $\gamma$ transitions (schematically shown in Fig. 16) with intensities of together $1.7 \%$ of the intensity within the SD band [84]. In this case, the SD band is observed in the spin range $24^{+}-62^{+}$. The present understanding of the decay-out mechanism of SD bands in the heavy mass regions is that it is a statistical process governed by the weak mixing of SD states with one or more of the adjacent closely spaced "hot" levels in the normal well separated by a potential energy barrier. The decay than occurs through the admixed component of the normal deformed state in the SD state wave function. This statistical picture implies that the transition intensity fragments into so many weak branches that a direct identification of the linking transitions is possible only for the strongest ones. Still today, only for a few of the many known superdeformed bands in the mass 150 and 190 regions excitation energies and spins are firmly assigned. Much more systematic information is necessary in order to address such issues as (i) the magnitude of shell corrections at large deformation, (ii) the particle configuration of the bands, (iii) the origin of identical SD bands and (iv) the mechanisms responsible for the sudden decay-out of the SD bands.

After the first pioneering experiments in very heavy nuclei, superdeformation has been shown to be a widespread phenomenon across the nuclidic chart. Besides the $A=150$ and $A=190$ mass regions discussed above, strongly deformed rotational bands in the second minimum have been observed since then in many other nuclei around $A \sim 130$, $\mathrm{A} \sim 80, \mathrm{~A} \sim 60$ and more recently also in the $\mathrm{A} \sim 40$ region. In the twenty years after the discovery of superdeformation in 1986 more than 250 discrete SD bands all over the chart of nuclides were identified. In addition, several excited superdeformed rotational bands were observed through the study of the quasi-continuum part of the $\gamma$-ray spectra [85]. In-beam studies of light to medium mass $\mathrm{N} \sim \mathrm{Z}$ nuclei are more challenging due to (i) the large number of different residual nuclei produced in the reactions (up to some 30), (ii) relatively large $\gamma$-ray energies and therefore low detection efficiencies and (iii) considerable Doppler broadening caused by the relatively high velocities and the large angle spread of the recoils induced by charged-particle emission. Only the powerful combination of highly efficient $4 \pi$ Ge detector arrays and ancillary detector systems, especially charged particle and neutron detectors, enabled the great progress in this field. Interestingly, superdeformation shows different aspects in the different mass regions. The $N=Z$ nuclei around doublymagic ${ }^{56} \mathrm{Ni}$ and ${ }^{40} \mathrm{Ca}$ are of special interest for the following reasons: (i) spherical and superdeformed magic numbers appear at similar particle numbers leading to dramatic examples of shape coexistence and (ii) these nuclei are amenable to different theoretical treatments that include shell model calculations in large configuration spaces, quantum Monte Carlo shell model descriptions and a va- riety of mean field approaches. As example, we will briefly discuss the superdeformed band in the $N=Z$ nucleus ${ }^{60} \mathrm{Zn}$, which represents the $N=Z=30$ "doubly magic" superdeformed core in the mass 60 region. In contrast to for example the mass 150 region, in which the nuclei of interest can be populated with large cross sections in fusion-evaporation reactions but the intensity of the SD bands accounts for only about $1 \%$ of the channel, interestingly ${ }^{60} \mathrm{Zn}$ is produced with only $\sim 0.1 \%$ of the total fusion cross section in the reaction ${ }^{40} \mathrm{Ca}\left({ }^{28} \mathrm{Si}, 2 \alpha\right)$ at 125 $\mathrm{MeV}$ but here the SD band is populated with about $60 \%$ of the channel intensity [86]. A superdeformed band consisting of eleven $\gamma$ transitions in the energy range 1.1-3.7 $\mathrm{MeV}$ was observed. Two one-step linking transitions into the ground state band as well as two additional two-step cascades have been identified allowing for a firm energy and spin assignment of the SD band. There are two observations which differ considerably from what we have seen in heavy nuclei. First, the identified linking transitions account for a significant fraction (about 37\%) of the SD intensity and second they are of stretched E2 character instead of $E 1$ as expected in a statistical decay process. Though a simple explanation for this observation is the suppression of isoscalar dipole transitions in $N=Z$ nuclei, the fact that the discrete decay-out scheme of the yrast SD band in the $N=Z+1$ nucleus ${ }^{59} \mathrm{Cu}$ is also dominated by stretched $E 2$ transitions indicates that this is not the full truth. Although still most of the decay-out intensity is fragmented over a large number of weak multistep pathways, some of the observed linkings have E2 transition strengths of up to 2 W.u., which is much too large to be explained within a purely statistical decay-out model used in the heavier mass regimes. Of course, the different level densities may play an important role, although the phase space, i.e. the excitation energy of the superdeformed states in the region of the decay-out, is comparable for the $A \sim 60$ and the $A \sim 190$ regions. An additional explanation for the seemingly selective decay-out pattern in the mass 60 region arises from the fact that the configurations of superdeformed and spherical states differ only by a relatively small number of nucleons while in heavier nuclei SD and ND configurations differ by the rearrangement of a substantial number of nucleon pairs. This fact hints at the inclusion of pairing in an appropriate decayout model. More theoretical effort is certainly needed in order to understand the decay-out mechanism in this mass region and relate it to the patterns observed in other mass regions.

The probably most spectacular result of the studies in the $\mathrm{A} \sim 60$ region has been the discovery of the unique new decay mode of prompt discrete-energy proton and $\alpha$ decays from high-spin states located in the strongly or superdeformed minimum of the nuclear potential into daughter states of spherical shapes. The particle decays compete with the conventional $\gamma$ decay-out. The first case in which such a decay-out via charged particle emission has been observed was ${ }^{58} \mathrm{Cu}$ populated in the reaction ${ }^{28} \mathrm{Si}\left({ }^{36} \mathrm{Ar}, \alpha \mathrm{pn}\right){ }^{58} \mathrm{Cu}[87]$. As illustrated in Fig. 18 a strongly deformed band $\left(\beta_{2} \sim 0.37\right)$ has been observed in this nu- 


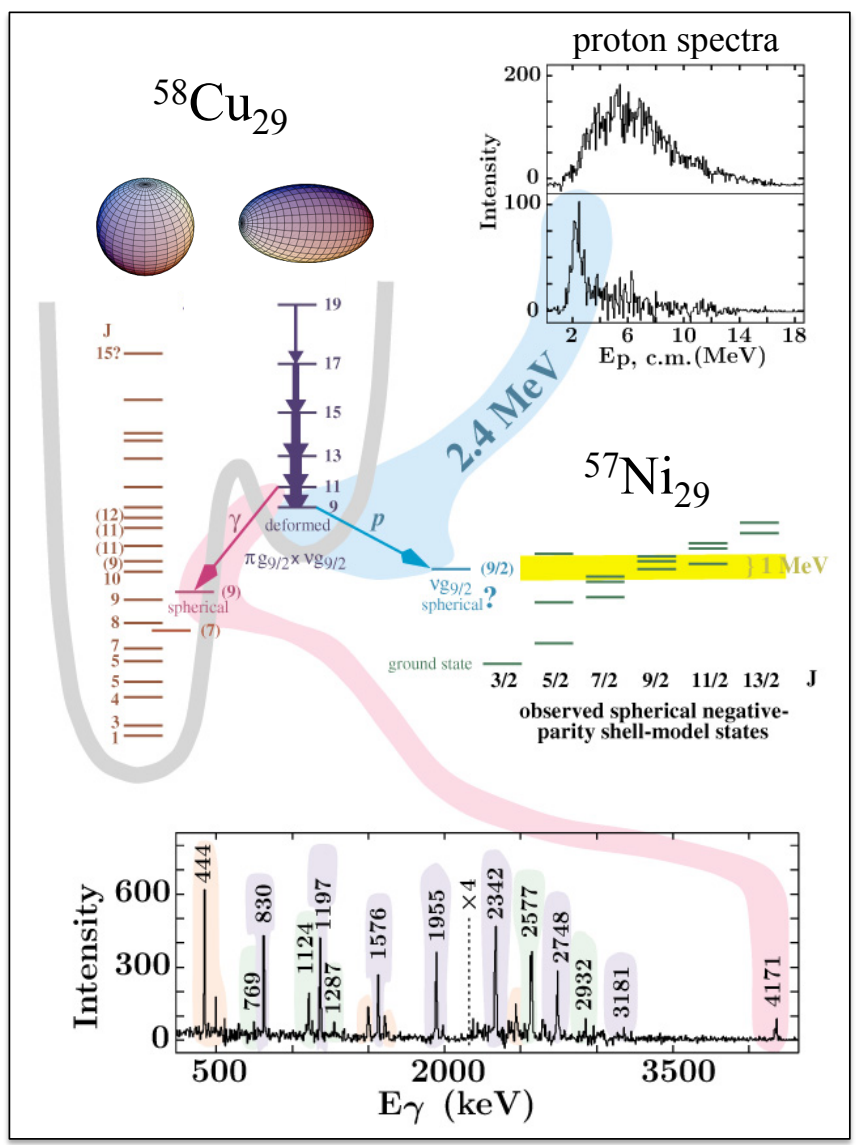

Fig. 18. Illustration of the discovery of prompt discrete proton emission from an excited state in the second minimum of ${ }^{58} \mathrm{Cu}$. The proton and $\gamma$-ray energy spectra prove the existence of two parallel decay branches out of the deformed minimum, via $\gamma$ decay to the spherical minimum of ${ }^{58} \mathrm{Cu}$ on one hand side and via proton emission to a spherical excited state in the isotone ${ }^{57} \mathrm{Ni}$ on the other. Figure adopted from Ref. [87].

cleus consisting of seven stretched quadrupole transitions (purple in Fig. 18). This band is linked to the spherical part of the level scheme via a $4.171 \mathrm{MeV} \gamma$ transition (pink in Fig. 18). This is the expected behaviour. The surprise is that requesting a coincidence with the lowest member of the deformed band, namely the $\gamma$ ray with $830 \mathrm{keV}$, besides the in-band transitions some $\gamma$ rays known to belong to the isotone ${ }^{57} \mathrm{Ni}$ are observed (green in Fig. 18). These data indicate that the $8915 \mathrm{keV}$ band head of the strongly deformed band in ${ }^{58} \mathrm{Cu}$ decays predominantly via proton emission into the spherical $3701 \mathrm{keV}$ level in the daughter nucleus ${ }^{57} \mathrm{Ni}$. This conclusion was confirmed through a careful examination of the proton energy spectra. In coincidence with $\gamma$ rays emitted from excited states in the first minimum, the protons show a broad energy distribution characteristic of protons evaporated in the fusionevaporation reaction (remember that ${ }^{58} \mathrm{Cu}$ was populated as $\alpha$ pn reaction channel). In contrast, in coincidence with the $830 \mathrm{keV}$ transition connecting excited states in the deformed second minimum, a sharp peak at 2.4(1) $\mathrm{MeV}$ is observed. This is the energy expected taking into account the binding energy difference between ${ }^{58} \mathrm{Cu}$ and ${ }^{57} \mathrm{Ni}$ as well as the transition energies of the observed $\gamma$ rays. A closer inspection reveals that only about $3 \%$ of the deexcitation of the $8915 \mathrm{keV}$ states proceeds via $\gamma$ decay, whereas proton emission accounts for the remaining $97 \%$. Since the discovery of this new mode of prompt discrete proton decay from excited states in the second minimum several other cases have been observed in neighbouring nuclei, among them also the doubly-magic nucleus ${ }^{56} \mathrm{Ni}[88]$. Furthermore, soon after the first proton decay in ${ }^{58} \mathrm{Cu}$, also a first case of prompt $\alpha$ decay has been identified, namely from a deformed band in ${ }^{58} \mathrm{Ni}$ into the spherical $6^{+}$yrast daughter state in ${ }^{54} \mathrm{Fe}$ [89]. In subsequent experiments the level scheme of ${ }^{58} \mathrm{Ni}$ was then significantly extended including now at least 14 discrete particle decays, protons and $\alpha$ particles, into excited states of the daughter nuclei ${ }^{57} \mathrm{Co}$ and ${ }^{54} \mathrm{Fe}[90]$. The discussed particle decays are two-dimensional quantum tunneling processes, in the course of which the remaining nuclear mean field is rearranged dramatically. Quantum-mechanical tunneling is a wide-spread phenomenon in the natural sciences. Therefore, a full understanding of this process may be of importance far beyond nuclear physics.

In the last paragraph we already mentioned, quite incidentally, that proton emission from a deformed rotational band was also observed in the doubly-magic nucleus ${ }^{56} \mathrm{Ni}$. Even two well-deformed rotational bands have been identified in this nucleus, one of them decaying via proton emission to the ground state of ${ }^{55} \mathrm{Co}$ [88]. Largescale shell model calculations have shown to be able to simultaneously describe the spherical excited states and the first rotational band within the $p f$ shell, the rotational band being built upon a four-particle four-hole (4p4h) excitation. This example nicely illustrates that even a doubly-magic nucleus can assume a deformed shape at not too high excitation energy and that furthermore these deformed structures can be described in the frame of the spherical shell model.

An even more impressive example for shape coexistence in a doubly-magic nucleus is the lighter self-conjugate nucleus ${ }^{40} \mathrm{Ca}$. This is a very special case for the simple reason that for particle number 20 energy gaps are predicted to exist not only for spherical shape, but also for different prolate and oblate deformations. In the single-particle diagram shown in Fig. 19, in addition to the spherical gap, two prolate and one oblate deformed shell gaps are present at $\beta_{2}$ values around $-0.4,0.3$, and 0.6 , respectively. Two rotational bands, built on the first two excited $0^{+}$states, were indeed observed in this nucleus by Ideguchi and coworkers, who populated the excited states of this nucleus using the reaction ${ }^{28} \mathrm{Si}\left({ }^{20} \mathrm{Ne}, 2 \alpha\right){ }^{40} \mathrm{Ca}$ [91]. A partial experimental level scheme of ${ }^{40} \mathrm{Ca}$ is shown in Fig. 19. In order to determine the deformation of these bands transition quadrupole moments were deduced from the measured fractional Doppler shifts of all in-band transitions as a function of the $\gamma$-ray energy. Assuming a rigid axially symmetric rotor these measured quadrupole moments correspond to quadrupole deformations of $\beta_{2} \sim 0.27$ for the band built on the first excited $0^{+}$state (blue in Fig. 19) 


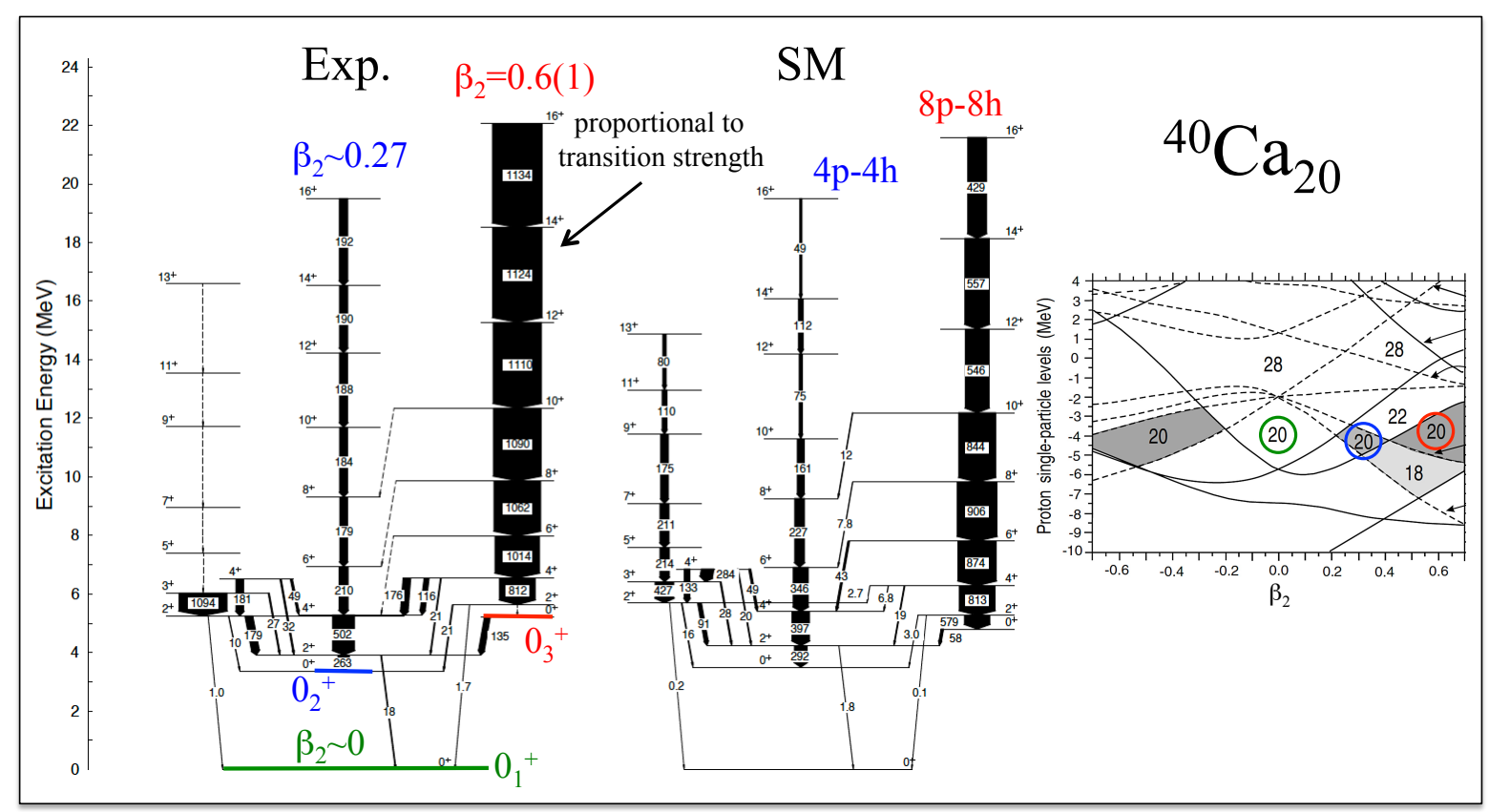

Fig. 19. Comparison between the experimentally established (left) and calculated (right) excitation scheme (partial) of the doubly-magic nucleus ${ }^{40} \mathrm{Ca}$ (taken from Refs. [91,92]). See text for details.

and $\beta_{2}=0.6(1)$, i.e. superdeformed shape, for that on top of the second excited $0^{+}$level (red in Fig. 19). In Ref. [91] the features of the two bands were explained by cranked relativistic mean field calculations to arise from $4 \mathrm{p}-4 \mathrm{~h}$ respectively $8 \mathrm{p}-8 \mathrm{~h}$ excitations.

Recently, an attempt was made to describe the dramatic example of shape coexistence observed in ${ }^{40} \mathrm{Ca}$ within the spherical shell model considering a valence space consisting of two major shells, namely the $p f$ and the $s d$ shell, and allowing for the creation of up to eight particle-hole pairs, i.e. excitations of nucleons from the $s d$ to the $p f$ shell [92]. The result of this calculation is compared to the experimental excitation scheme in Fig. 19. As can be seen not only the excitation energies, but also the transition strengths within the two rotational bands are nicely reproduced by the shell model calculations. As in the case of the mean field calculations mentioned above also the SM assigns $4 \mathrm{p}-4 \mathrm{~h}$ respectively $8 \mathrm{p}-8 \mathrm{~h}$ character to the normal deformed and superdeformed bands built on the excited $0^{+}$states. This example illustrates in an impressive way that with the exploration of the excitation energy versus spin plane, a field which was initiated in the 1970s with the discovery of the backbending phenomenon, the thinking in fixed categories, e.g. the shell model is used to describe spherical nuclei close to the shell closures and the collective models are used to describe excitations of deformed nuclei in the open shells, had to be abandoned.

\subsection{The future of $\gamma$-ray spectroscopy}

In the last section we tried to give an idea about the richness of phenomena which were observed studying the atomic nucleus at high excitation energy and high angular momentum. Heavy-ion induced fusion-evaporation reactions were shown to be an efficient tool to populate nuclei at spins as high as $70 \hbar$ and excitation energies up to $35 \mathrm{MeV}$. The sensitivity of the experiment then mainly depends on the efficiency of the $\gamma$-ray spectrometer used to detect the long cascades of $\gamma$ rays emitted in the deexcitation of these states towards the ground state. How closely the progress in the exploration of the $\mathrm{E}_{x}$ vs. I plane is related to the advancement in instrumentation becomes evident when considering the case of ${ }^{158} \operatorname{Er}$ (see Ref. [93] for details). As mentioned in Section 5, this nucleus was one of the first cases in which Coriolis-induced pair breaking (backbending) was discovered in 1972 [76] and the first in which a second alignment was observed [79]. The first alignment of two $i_{13 / 2}$ neutrons at spin $\sim 14 \hbar$ and the second alignment of two $h_{11 / 2}$ protons at spin $\sim 28 \hbar$ were illustrated already in Fig. 15 and are shown in Fig. 20 again, this time in the $\mathrm{E}_{x}$ vs. I plane. In the 1980s the TESSA and HERA arrays of Ge detectors enabled the observation of a dramatic change of structure around spin $38 \hbar$. Less collective band structures, which reach high spin by aligning the single-particle angular momenta of the valence nucleons outside the ${ }^{146} \mathrm{Gd}_{82}$ core, become energetically favoured. The alignment of the valence particles in these bands causes the shape of the nucleus to become oblate. These bands are called terminating bands because they terminate once all valence particles are aligned to the maximum available spin, in the case of ${ }^{158} \mathrm{Er}$ in the range 46-49 . This case can actually be considered a textbook example of the phenomenon of band termination in heavy nuclei. However, the story of ${ }^{158} \mathrm{Er}$ does not stop here. To generate even higher angular momentum the ${ }^{146} \mathrm{Gd}$ core 


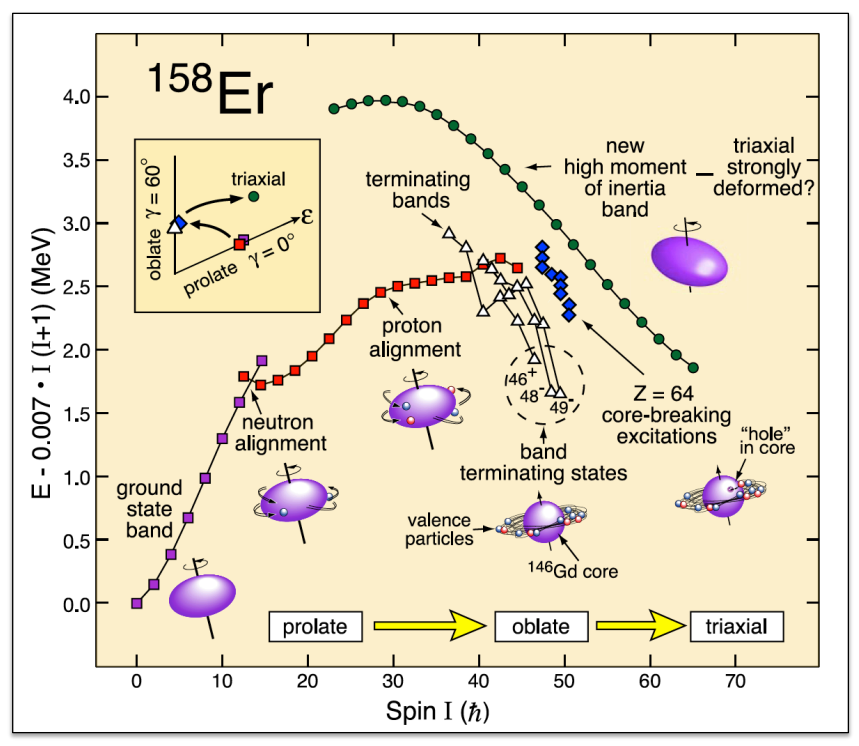

Fig. 20. Evolution of the nuclear structure of ${ }^{158}$ Er with spin. Excitation energies of a variety of observed structures are plotted with respect to a rigid-rotor reference in order to emphasize the changes that occur along and close to the yrast line. The strongest new high moment-of-inertia band is included, but its exact excitation energy is not known. The inset illustrates the changing shape of ${ }^{158} \mathrm{Er}$ with increasing spin within the standard $(\epsilon, \gamma)$ deformation plane (from Ref. [93]).

has to be broken which costs about $1 \mathrm{MeV}$ of energy at $50 \hbar$. Some weak high-energy $\gamma$ rays decaying from states beyond band termination into the band-terminating states have been observed in 2006 using the Gammasphere $\gamma$-ray spectrometer [94]. The so far last chapter in the saga of ${ }^{158} \mathrm{Er}$ was written shortly after with the identification of four rotational structures, displaying high moments of inertia, which extend up to spin $65 \hbar$ and bypass the bandterminating states discussed above. These bands imply the return of collective rotation at ultrahigh spin. Comparison with cranked Nilsson-Strutinsky calculations suggests that these sequences are most likely strongly deformed triaxial structures. It is an amazing feature that the nucleus ${ }^{158} \mathrm{Er}$ exhibits a variety of different shapes in different regions of the $\mathrm{E}_{x}$ vs. I plane, from prolate to oblate and further to triaxial deformation, and all this during the few picoseconds between the population of the highly excited nucleus until it reaches its ground state. Note that the strongly deformed bands carry only about $10^{-5}-10^{-4}$ of the channel intensity. With the observation of these bands the sensitivity limit of instruments such as Gammasphere and EUROBALL is reached (compare Fig. 17). To proceed and to fully explore the spin regime until fission sets in, the development of new, even more powerful $\gamma$-ray spectrometer is necessary.

Using $\gamma$-ray spectrometer built out of escape-supressed Ge detectors the photopeak efficiency is limited to about $10 \%$ (the efficiency reached with Gammasphere and EUROBALL) due to the simple fact that independent on the exact geometry a large fraction of the total solid angle will always be covered by collimators to prevent the radiation from the target to directly enter the compton-suppression (CS) shields. This problem of solid angle covered by dead material is illustrated in Fig. 21 which shows the view from the target position into one half of the EUROBALL spectrometer. The only way to overcome this hindrance is the use of a Ge shell, i.e. an instrument consisting exclusively of Ge crystals. Monte Carlo simulations show that in this way photopeak efficiencies of $40-60 \%$ can be reached. However, the problem of the Ge shell is to distinguish between two $\gamma$ rays emitted from the target and detected in two adjacent detectors and one $\gamma$ ray emitted from the target and Compton scattered from one to the other detector. The only solution to this problem is to increase the number of Ge detectors in the shell to 1000 or more in order to reduce the probability that two coincident $\gamma$ rays from a high multiplicity cascade interact with neighbouring detectors. Unfortunately, such a solution is unrealistic due to its extremely high cost. A new perspective then opened up with the development of the first segmented Ge detectors in the beginning of this century. Position-sensitivity of the detectors is achieved by both segmentation of the outer contact and by analyzing the charge drift times within a segment and the mirror charges induced in the neighbouring segments. Using digital electronics and pulse-shape analysis techniques it is possible to identify the energy, time and position of every interaction point of a $\gamma$ ray as it interacts, scatters and is finally absorbed in the $4 \pi$ ball. The full event can be reconstructed offline using algorithms based on the KleinNishina formula.

This is the new concept in array design, based on $\gamma$-ray tracking in highly segmented germanium detectors (see Fig. 21). Besides the increase in efficiency there is another aspect which makes $\gamma$-ray tracking arrays very attractive. The reconstruction of the position of the first interaction point in the segmented Ge crystal allows for a very precise determination of the angle of $\gamma$ emission relative to the beam axis. As a consequence, a much better Doppler correction can be achieved as compared to the use of un-

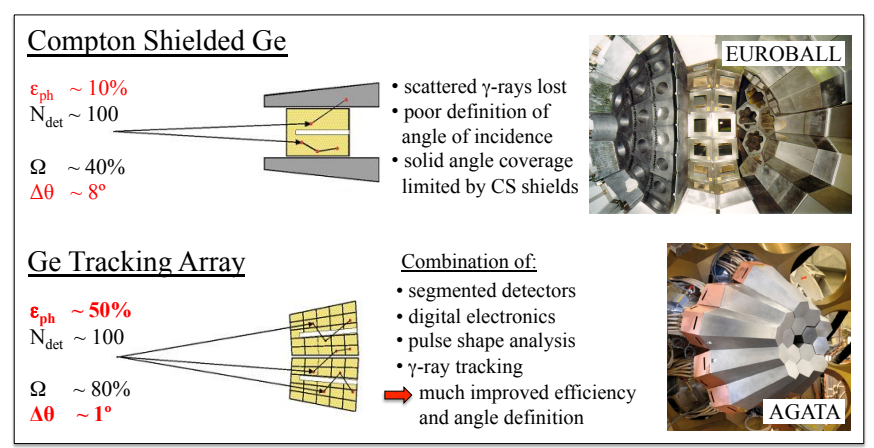

Fig. 21. Comparison between conventional $4 \pi \gamma$-ray spectrometer consisting of unsegmented Ge crystals such as EUROBALL and the future tracking arrays such as AGATA. The use of segmented Ge detectors in combination with digital electronics, pulse shape analysis and tracking algorithms leads to a much improved efficiency and sensitivity. 
segmented detectors whenever the $\gamma$ rays are emitted from a moving nucleus. This is particularly important for $\gamma$-ray spectroscopy using fast radioactive ion beams. The concept of a tracking arrays was first tested and successfully demonstrated by smaller arrays of segmented detectors such as the SeGA array at NSCL [95], the MINIBALL at ISOLDE [96] or the TIGRESS array at TRIUMF (Vancouver, Canada) [97].

Using highly segmented Ge crystals a $4 \pi$ Ge shell can be realized which consists of only 100-200 instead of the 1000 individual detectors needed in the case of unsegmented crystals as mentioned above. Monte Carlo simulations show that with this reduced number of crystals (and consequently reduced cost) such an array would still be able to combine a very high photopeak efficiency (maximum coverage of the solid angle with Ge detectors) with an excellent ability to correct for Doppler effects and a very good peak-to-total ratio (by distinguishing between fully and partially absorbed events). As in the late 1980s, when the Gammasphere and EUROBALL projects were launched in parallel by American and European collaborations, respectively, two projects aiming for the construction of $4 \pi$ tracking arrays are currently under way. The American project is called GRETA (Gamma Ray Energy Tracking Array) and in Europe scientists from twelve different countries are working on the AGATA (Advanced GAmma Tracking Array) project. Although the design of the two instruments is basically very similar, in detail different technical solutions are chosen. For a detailed discussion of the technical aspects of these projects see Ref. [98, 99].

\section{Outlook}

In this article I have discussed some recent achievements in the field of nuclear structure physics. I tried to demonstrate that within the last few decades, the limits of our knowledge of the atomic nucleus have been pushed out tremendously in two different directions. First, it became feasible to study nuclei far-off stability at the limits of nuclear binding, i.e. in the regions of extreme isospin and extreme masses. These studies lead to the discovery of surprising phenomena such as neutron halos and skins on the neutron-rich side as well as the first observation of two-proton radioactivity and spectroscopic studies behind the proton dripline on the proton-rich side. In particular, the study of light neutron rich nuclei taught us that part of our established knowledge about the atomic nucleus, gained over many years studying nuclei close to the valley of stability, may have to be revised in regions far-off stability. In particular, the concept of a static shell structure with global magic numbers all over the chart of nuclei had to be given up in favor of a more dynamic view of shell evolution, in which classical magic numbers can disappear in certain regions of nuclei while new shell gaps can arise. On the other hand, atomic nuclei have been investigated under exotic conditions such as very high temperature or high rotational frequency. These studies enabled the discovery of exotic shapes in nuclei, e.g. superdeformation and triaxiality, new decay modes such as prompt charged particle emission from excited states in the second minimum as well as the study of shape coexistence, phase transitions and new modes of collective excitations such as the wobbling mode [100].

The field of nuclear physics research has undoubtedly experienced a renaissance during recent years and all the exciting results stimulate appetite for more. Currently, the next generation of high intensity radioactive beam facilities using both in-flight and ISOL techniques is in the planning stage both in the US and in Europe (FAIR, FRIBS, EURISOL etc.). These facilities will provide radioactive ion beams over a wide energy range and with intensities much higher than available at the moment. This will not only allow to establish the limits of nuclear existence for heavier elements up to Manganese $(Z=25)$ but the higher intensities for neutron-rich nuclei will enable in addition the measurement of basic properties such as masses and lifetimes which are crucial e.g. for our understanding of astrophysical processes. For many isotopes the available intensities will be high enough to perform secondary reactions with the radioactive beams, e.g. fusionevaporation reactions or transfer reactions. Looking at the proton-rich side of the nuclear chart, it will for the first time be possible to map the proton drip line for mediummass odd- $Z$ nuclei and to identify ground state proton emitters up to $Z=93$. Concerning the heaviest nuclei, the synthesis of new species may become possible in the fusion of re-accelerated neutron-rich radioactive beams with ${ }^{208} \mathrm{~Pb},{ }^{238} \mathrm{U}$ or ${ }^{248} \mathrm{Cm}$ targets. The exploration of these neutron-rich superheavy nuclei furthermore would help to put the identification of SHE created in hot fusionevaporation reactions with stable beams on a solid footing.

Of course, parallel to the development of more intense radioactive beam facilities the current instrumentation has to be improved. One example is the development of an improved fragment separator "Super-FRS" at GSI, another one new $\gamma$-ray spectrometer for the use at radioactive beam facilities. Even though radioactive beams from the next generation facilities will often approach today's intensity of stable beams, the most exotic nuclei under investigation will always be produced with extremely low rates. A $\gamma$-ray spectrometer to study these nuclei must be a universal instrument that fulfills some strict criteria. The best solution seems to be a $4 \pi$ shell of large segmented Ge crystals in which the individual interaction points of the $\gamma$ quanta are disentangled numerically in order to allow for an efficient Doppler correction. Both in the US and Europe such tracking arrays are currently under development.

In conclusion, everything is prepared for a very exciting future of nuclear physics research and we are looking forward to many more surprises the atomic nucleus is certainly still keeping for us. 
This work was supported by the Spanish Ministerio de Economía y Competitividad under contract FPA201457196-C5-4-P.

\section{References}

1. M. Goeppert-Mayer, Phys. Rev. 74, (1948) 235; Phys. Rev. 75, (1949) 1969; Phys. Rev. 78, (1950) 16.

2. I. Tanihata et al., Phys. Rev. Lett. 55, (1985) 2676.

3. P.G. Hansen and B. Jonson, Europhys. Lett. 4, (1987) 409.

4. G. Huber et al., Phys. Rev. C18, (1978) 2342.

5. T. Suzuki et al., Phys. Rev. Lett. 75, (1995) 3241.

6. T. Baumann et al., Phys. Lett. B439, (1998) 256.

7. D. Cortina-Gil et al. Eur. Phys. J. A10, (2001) 49.

8. I. Tanihata, H. Savajols and R. Kanungo, Prog. Part. Nucl. Phys. 68, (2013) 215.

9. H. Sakurai et al., Phys. Lett. B448, (1999) 180.

10. M. Notani et al., Phys. Lett. B542, (2002) 49.

11. T. Baumann et al., Nature 449, (2007) 1022.

12. A. Gade, Nuclear Physics News 23, (2013) 10.

13. L. Gaudefroy and S. Grevy, Nuclear Physics News 20, (2010) 13.

14. R. Kanungo, Phys. Scr. T152, (2013) 014002.

15. O. Sorlin and M.-G. Porquet, Phys. Scr. T152, (2013) 014003.

16. C. Thibault et al., Phys. Rev. C12, (1975) 644.

17. C. Detraz et al., Phys. Rev. C19, (1979) 164.

18. E.K. Warburton, J.A. Becker and B.A. Brown, Phys. Rev. C41, (1990) 1147.

19. T. Otsuka et al., Phys. Rev. Lett. 87, (2001) 082502.

20. T. Otsuka et al., Phys. Rev. Lett. 95, (2005) 232502.

21. R. Kanungo et al., Phys. Rev. Lett. 102, (1999) 152501.

22. H. Simon et al., Phys. Rev. Lett. 83, (1999) 496.

23. D. Steppenbeck et al., Nature 502, (2013) 207.

24. F. Wienholtz et al., Nature 498, (2013) 346.

25. M. Bender, P.-H. Heenen and P.-G. Reinhard, Rev. Mod. Phys. 75, (2003) 121.

26. T.R. Rodrguez and J.L. Egido, Phys. Rev. C81, (2010) 064323.

27. N. Lopez Vaquero, T.R. Rodriguez and J.L. Egido, Physics Letters B704, (2011) 520.

28. M. Borrajo, T.R. Rodriguez and J.L. Egido, Phys. Lett. B746, (2015) 341.

29. T.R. Rodriguez and J.L. Egido, Phys. Rev. Lett. 99, (2007) 062501.

30 .

31. J. Fridmann et al., Nature 435, (2005) 922.

32. B. Bastin et al., Phys. Rev. Lett. 99, (2007) 022503.

33. S. Takeuchi et al., Phys. Rev. Lett. 109, (2012) 182501.

34. G. Lorusso et al., Phys. Rev. Lett. 114, (2015) 192501.

35. K.-L. Kratz et al., Astrophys. J. 403, (1993) 216.

36. B. Chen et al., Phys. Lett. B355, (1995) 37.

37. S. Hofmann et al., Z. Phys. A305, (1982) 111.

38. O. Klepper et al., Z. Phys. A305, (1982) 125.

39. D. Seweryniak et al., Phys. Rev. Lett. 86, (2001) 1458.

40. M. Karny et al., Phys. Lett. B664, (2008) 52.

41. V.I. Goldansky, Nucl. Phys. 19, (1960) 482.

42. M. Pfützner et al., Eur. Phys. J. A14, (2002) 279.

43. J. Giovinazzo et al., Phys. Rev. Lett. 89, (2002) 102501.

44. M.D. Cable et al., Phys. Rev. Lett. 50 (1983) 404.

45. B. Blank and M.J.G. Borge, Prog. Part. Nucl. Phys. 60 (2008) 403.
46. O.V. Bochkarev et al., Sov. J. Nucl. Phys. 55, (1992) 955.

47. R.A. Kryger et al., Phys. Rev. Lett. 74, (1995) 860.

48. J. Giovinazzo et al., Phys. Rev. Lett. 99, (2007) 102501.

49. K. Miernik et al., Phys. Rev. Lett. 99, (2007) 192501.

50. M. Pfützner, M. Karny, L.V. Grigorenko and K. Riisager,

Rev. Mod. Phys. 84, (2012) 567.

51. A. Gadea et al., Phys. Rev. Lett. 97, (2006) 152501.

52. D. Rudolph et al., Phys. Rev. C 78, (2008) 121301(R).

53. S. Ceruti et al., Phys. Rev. Lett. 115, (2015) 222502.

54. M.A. Bentley and S.M. Lenzi, Prog. Part. Nucl. Phys. 59,

(2007) 497.

55. B. Cederwall et al., Nature 469, (2011) 68.

56. A.N. Andreyev et al., Nature 405, (2000) 430.

57. K. Heyde and J.L. Wood, Rev. Mod. Phys. 83, (2011) 1467.

58. J.L. Egido et al., Phys. Rev. Lett. 93, (2004) 082502.

59. A. Sobiczewski et al., Phys. Lett. 22, (1966) 500.

60. W.D. Myers and W.J. Swiatecki, Nucl. Phys. 81, (1966)

1.

61. S.G. Nilsson et al., Nucl. Phys. A131, (1969) 1.

62. S. Cwiok, P.-H. Heenen and W. Nazarewicz, Nature 433 (2005) 705.

63. Yu. Ts. Oganessian and V.K. Utyonkov, Rep. Prog. Phys. 78, (2015) 036301.

64. Yuri Oganessian, Nuclear Physics News. 23/1, (2013) 15.

65. K. Morita et al., J. Phys. Soc. Jpn 81, (2012) 103201.

66. Y.T. Oganessian et al., Phys. Rev. C69, (2004) 021601(R).

67. E. Minaya Ramirez et al., Science 337, (2012) 1207.

68. P. Reiter et al., Phys. Rev. Lett. 82, (1999) 509.

69. R.-D. Herzberg and P.T. Greenlees, Prog. Part. Nucl. Phys. 61, (2008) 674

70. P.T. Greenlees et al., Phys. Rev. Lett. 109, (2012) 012501.

71. S.G. Nilsson, Dan. Mat. Fys. Medd. 29, (1955) no 16.

72. R.-D. Herzberg et al., Nature 442, (2006) 896.

73. B. Gall and P.T. Greenlees, Nuclear Physics News. 23/3, (2013) 23.

74. H. Morinaga and P.C. Gugelot, Nuclear Physics 46, (1963) 210.

75. A. Johnson, H. Ryde and S.A. Hjorth, Nuclear Physics A179, (1972) 753 .

76. H. Beuscher et al., Phys. Lett. B40, (1972) 449.

77. F.S. Stephens and R.S. Simon, Nuclear Physics A183, (1972) 257.

78. B.R. Mottelson and J.G. Valatin, Phys. Rev. Lett. 5, (1960) 511.

79. I.Y. Lee et al., Phys. Rev. Lett. 38, (1977) 1454.

80. P. Twin et al., Phys. Rev. Lett. 57, (1986) 811.

81. D. Kleppner, Physics Today 44/12, (1991) 9.

82. J. Eberth and J. Simpson, Prog. Part. Nucl. Phys. 60, (2008) 283.

83. T.L. Khoo et al., Phys. Rev. Lett. 76, (1996) 1583.

84. T. Lauritsen et al., Phys. Rev. Lett. 88, (2002) 042501.

85. S. Leoni et al., Phys. Rev. Lett. 101, (2008) 142502.

86. C.E. Svensson et al., Phys. Rev. Lett. 82, (1999) 3400.

87. D. Rudolph et al., Phys. Rev. Lett. 80, (1998) 3018.

88. D. Rudolph et al., Phys. Rev. Lett. 82, (1999) 3763.

89. D. Rudolph et al., Phys. Rev. Lett. 86, (2001) 1450.

90. E.K. Johansson et al., Phys. Rev. C 80, (2009) 014321.

91. E. Ideguchi et al., Phys. Rev. Lett. 87, (2001) 222501.

92. A. Poves, International School on Exotic Beams, Santiago

de Compostela, 2010

93. E.S. Paul et al., Phys. Rev. Lett. 98, (2007) 012501.

94. M.A. Riley et al., Phys. Scr. T125, (2006) 123. 
95. W.F. Mueller et al., Nucl. Instr. Meth. A466, (2001) 492 65.

96. N. Warr et al., Eur. Phys. J. A49, (2013) 40.

97. H.C. Scraggs et al., Nucl. Instr. Meth. A543, (2005) 431.

98. S. Akkoyun et al., Nucl. Instr. Meth. A668, (2012) 26.

99. I.Y. Lee and J. Simpson, Nuclear Physics News. 20/4, (2010) 23.

100. S.W. Ødegård et al., Phys. Rev. Lett. 86, (2001) 5866. 